\title{
Modeling Fragrance Components Release from a Simplified Matrix Used in Toiletries and Household Products
}

\author{
Patrícia Costa*, Miguel A. Teixeira, Yohan Lièvre, José Miguel Loureiro, Alírio E. \\ Rodrigues
}

Laboratório Associado LSRE-LCM, Faculdade de Engenharia, Universidade do Porto, Rua Dr. Roberto Frias s/n, 4200-465 Porto, Portugal.

*E-mail: patriciasc@fe.up.pt. Tel.: +351 22041 3688. Fax: +351 225081674.

Supporting Information. Figures on the molecular structure of the odorants and matrix, schematic representation of the experimental procedures and headspace gas chromatography analysis, gas phase concentrations and odor intensities as a function of their liquid phase concentrations for the binary, ternary and quaternary fragrance mixtures, tables on the studied fragrance mixtures and total fragrance concentration percentages, UNIFAC groups and subgroups, number of groups in molecule, the parameters of molecular van der Waals volumes and surface area, theoretical and experimental Henry's law constants together with the coefficient of determination, $\mathrm{F}$ value, maximum liquid concentrations, and relative deviation of Henry's law constants between experimental and theoretical Henry's law constants for the studied mixtures, predicted activity coefficients, liquid phase and predicted molar composition of the vapor phase of the fragrance mixtures are provided.

This information is available free of charge via the Internet at http://pubs.acs.org. 
<smiles>C=C(C)[C@H]1CC=C(C)CC1</smiles>

Limonene

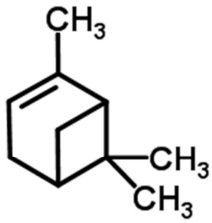

a-Pinene

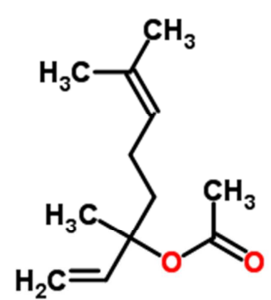

Linalyl acetate

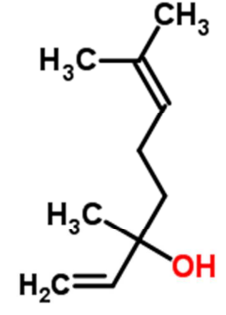

Linalool<smiles>Cc1ccc(C(C)C)cc1</smiles>

p-Cymene

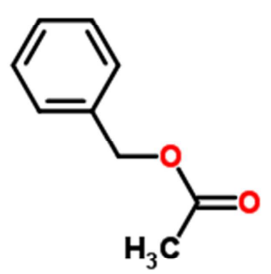

Benzyl acetate<smiles>CCOC(C)=O</smiles>

Ethyl acetate<smiles>CCC(O)OC(O)CC</smiles>

Dipropylene glycol

Figure S1. Molecular structure of the odorants and matrix studied in this work.

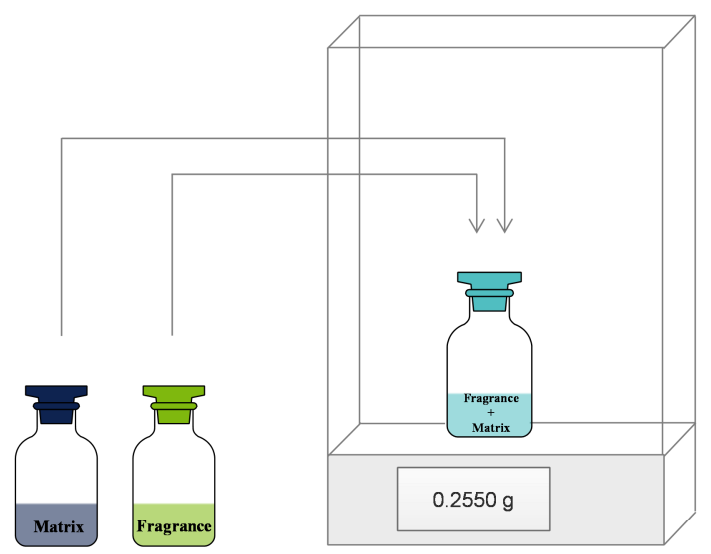

Step1: Liquid mixtures containing the fragrance and matrix at different concentrations were prepared gravimetrically in $4 \mathrm{~mL}$ vials using an analytical balance.

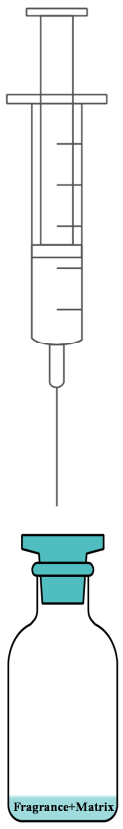

Step 2: Fragrance mixtures $(1 \mathrm{~mL})$ were placed in $20 \mathrm{~mL}$ closed-cap headspace vials and allowed to equilibrate for at least $24 \mathrm{~h}$, at $23^{\circ} \mathrm{C}$.
Step 3: $0.4 \mathrm{~mL}$ of the gas phase were collected and then injected in HS-GC $\$$ to evaluate the fragrance concentration.

Figure S2. Schematic representation of the experimental procedures taken for sample preparation and headspace sampling. 


\section{$\S_{\text {Headspace Gas Chromatography Analysis }}$}

The fragrance concentrations in the gas phase above the liquid mixtures were determined by headspace gas chromatography analysis using a Varian CP-3800 equipped with a split/splitless injector, FID detector, and a capillary column Chrompack CP-Wax 52 CB, $50 \mathrm{~m}$ length, $0.25 \mathrm{~mm}$ i.d., and $0.2 \mu \mathrm{m}$ film thickness. The injector and detector ports were set at 240 and $250{ }^{\circ} \mathrm{C}$, respectively. Injection volume for the gas phase was $0.4 \mathrm{~mL}$ and the split ratio was 10:1. Gas sampling and injection were performed using a gastight syringe from SGE (Australia) installed in an automatic headspace sampler HT250D by HTA SrL. The carrier gas used was helium (He N60) with a constant flow rate of $1 \mathrm{~mL} / \mathrm{min}$.
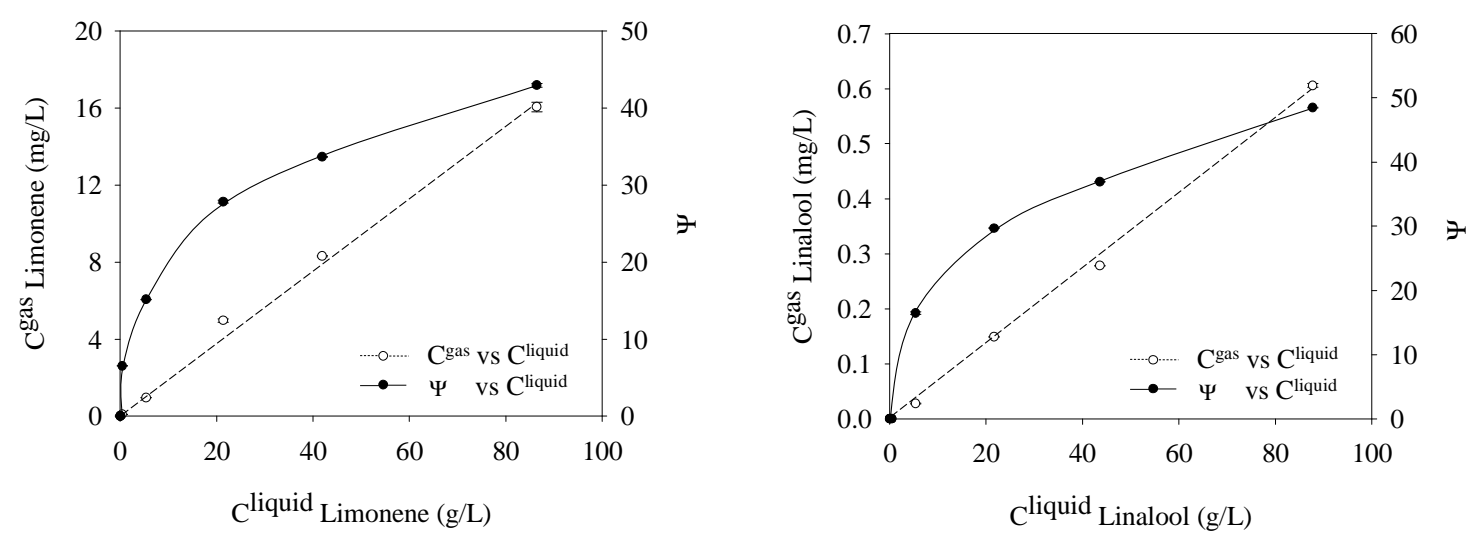

Figure S3. Gas phase concentrations and odor intensities (using the Stevens' power law as odor intensity model) as a function of their liquid phase concentrations for the binary fragrance mixture limonene + linalool diluted in DPG. 

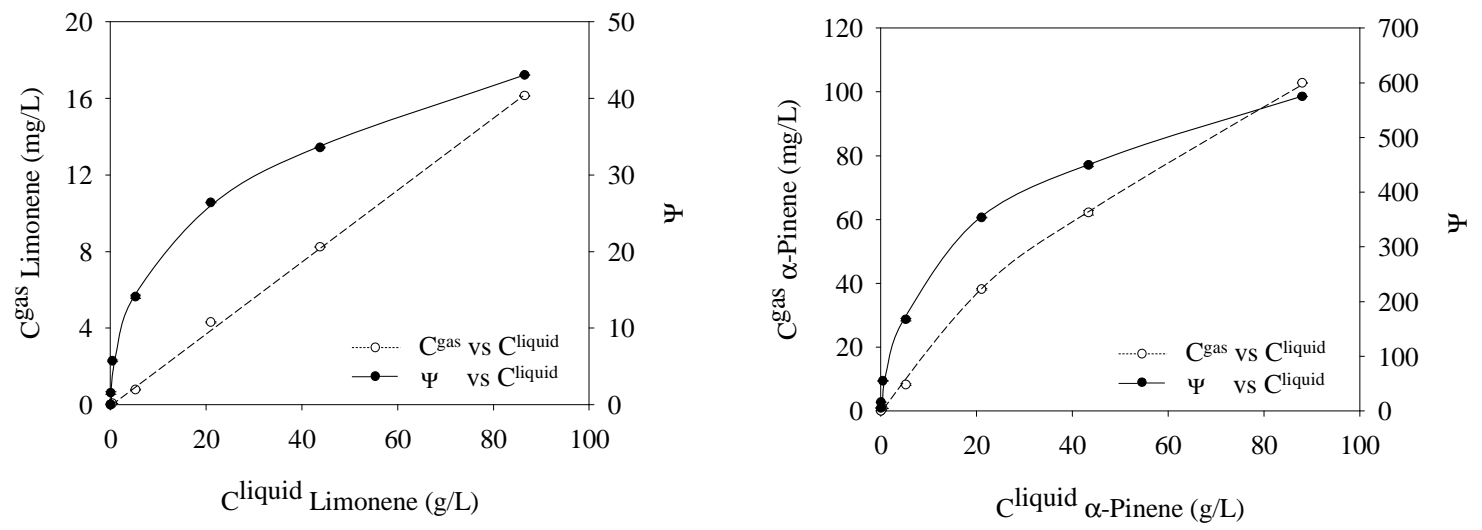

Figure S4. Gas phase concentrations and odor intensities (using the Stevens' power law as odor intensity model) as a function of their liquid phase concentrations for the binary fragrance mixture limonene $+\alpha$-pinene diluted in DPG.
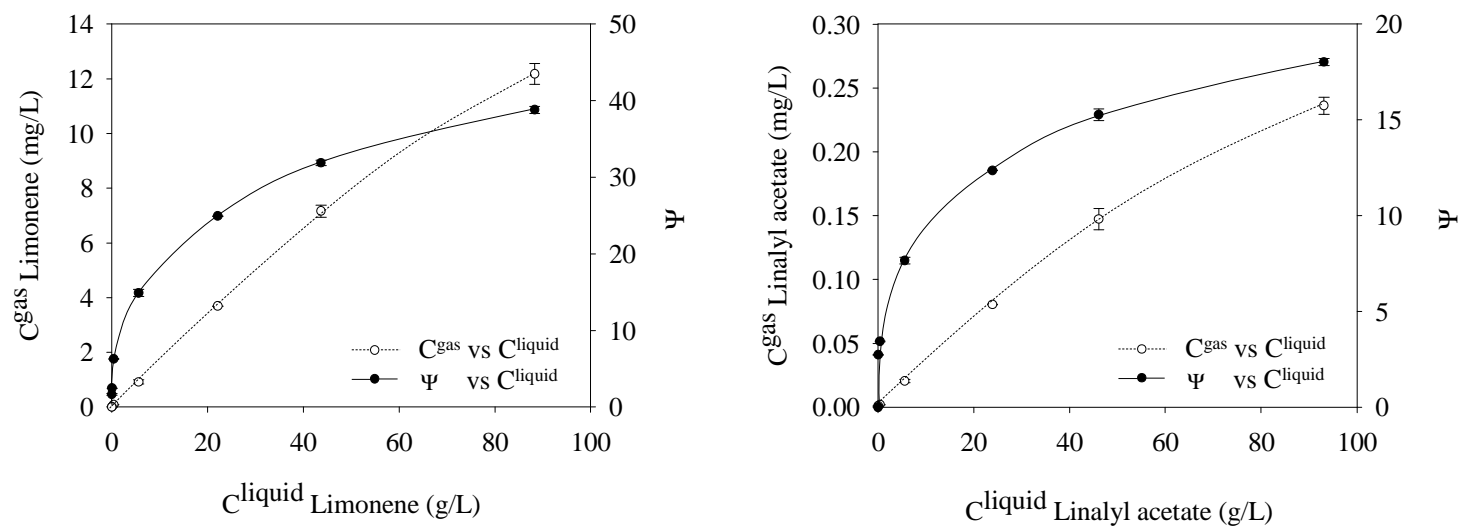

Figure S5. Gas phase concentrations and odor intensities (using the Stevens' power law as odor intensity model) as a function of their liquid phase concentrations for the binary fragrance mixture limonene + linalyl acetate diluted in DPG. 

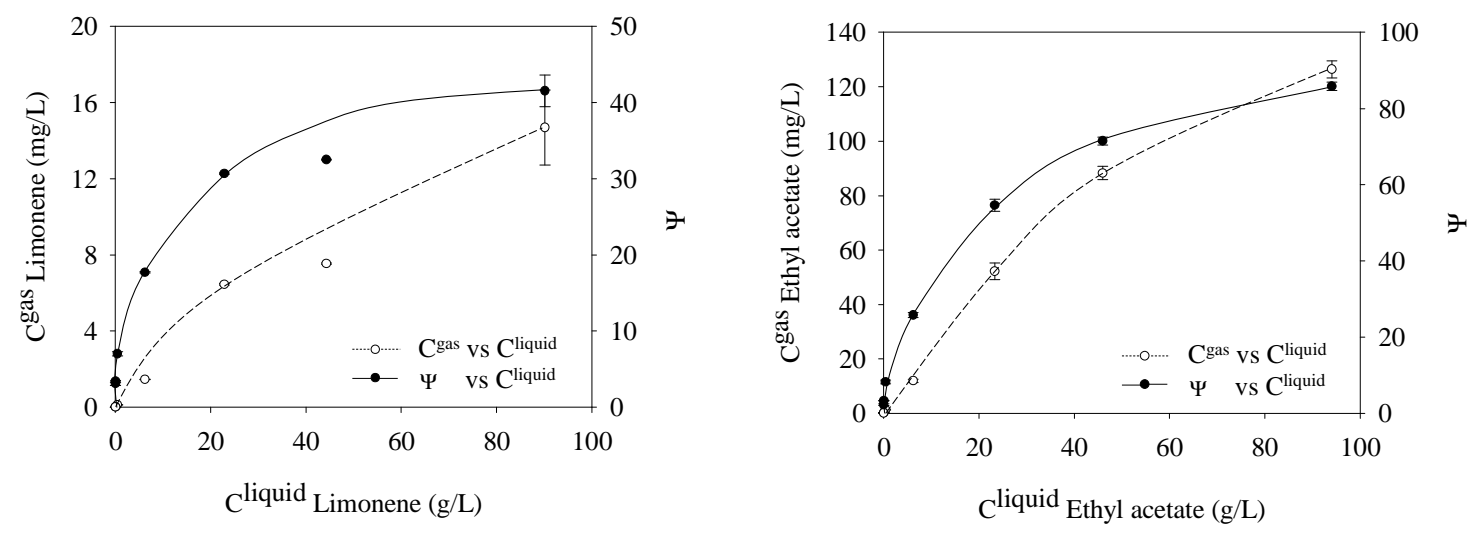

Figure S6. Gas phase concentrations and odor intensities (using the Stevens' power law as odor intensity model) as a function of their liquid phase concentrations for the binary fragrance mixture limonene + ethyl acetate diluted in DPG.
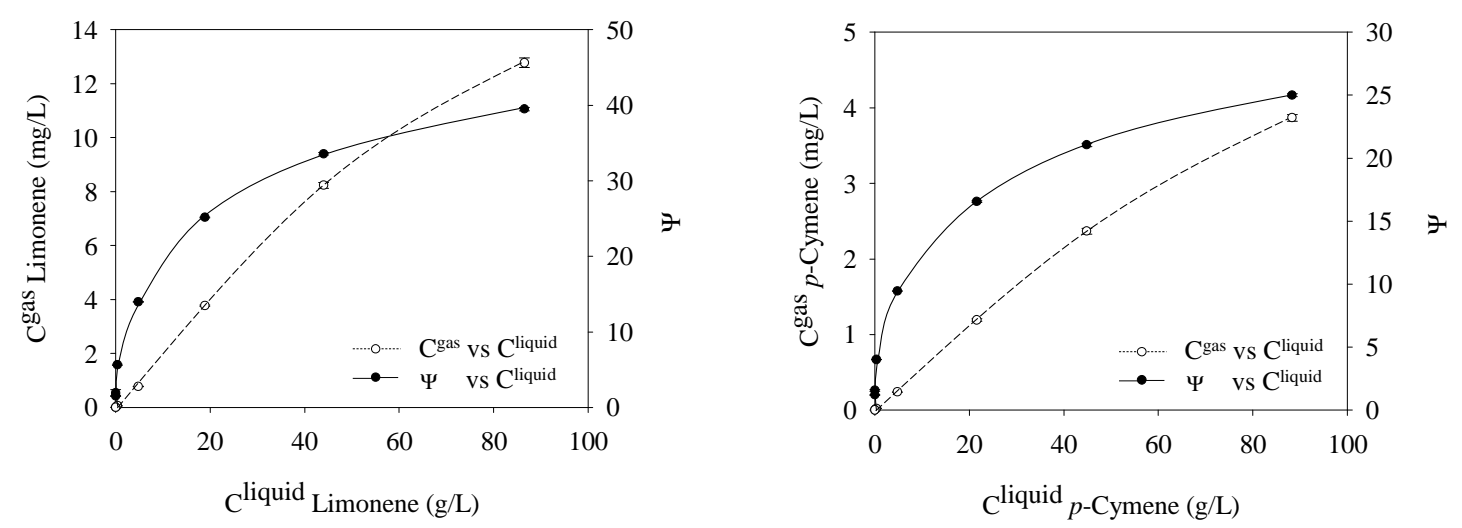

Figure S7. Gas phase concentrations and odor intensities (using the Stevens' power law as odor intensity model) as a function of their liquid phase concentrations for the binary fragrance mixture limonene $+p$-cymene diluted in DPG. 
a) Limonene $+\alpha$-Pinene+Linalyl acetate
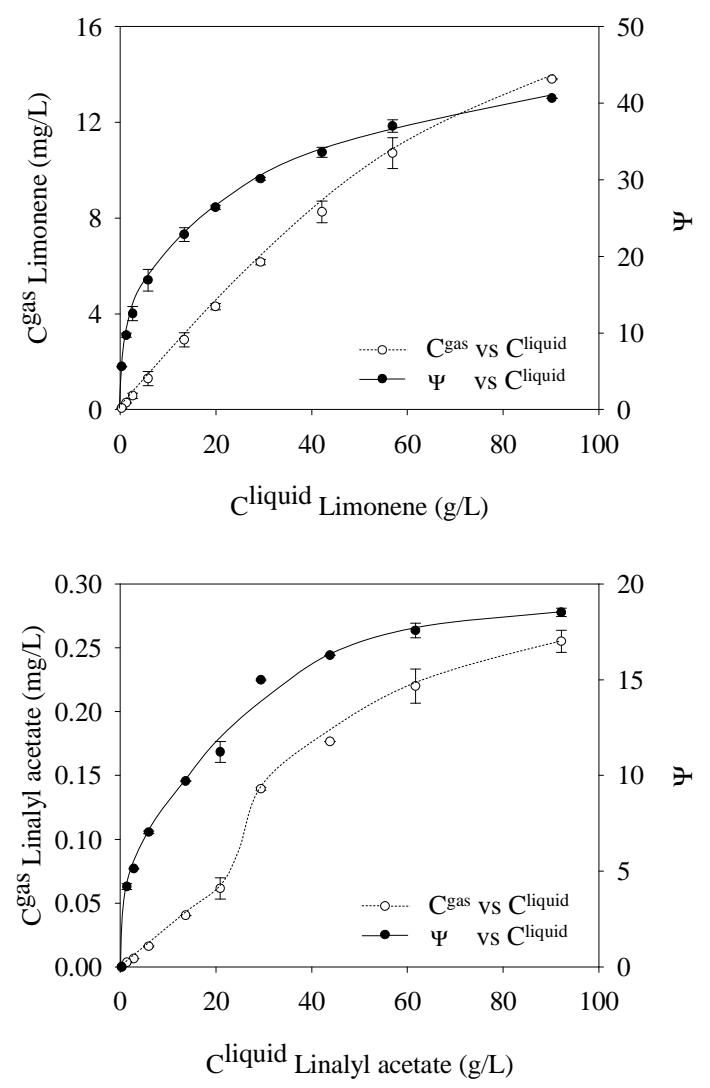

b) Limonene $+p$-Cymene+Linalool
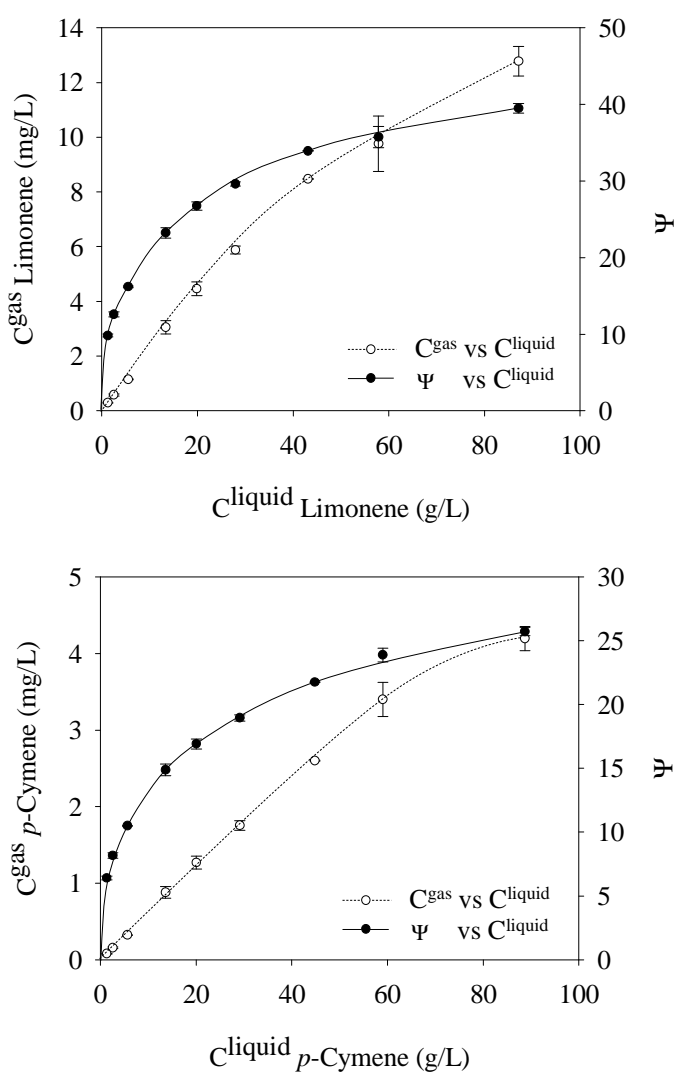
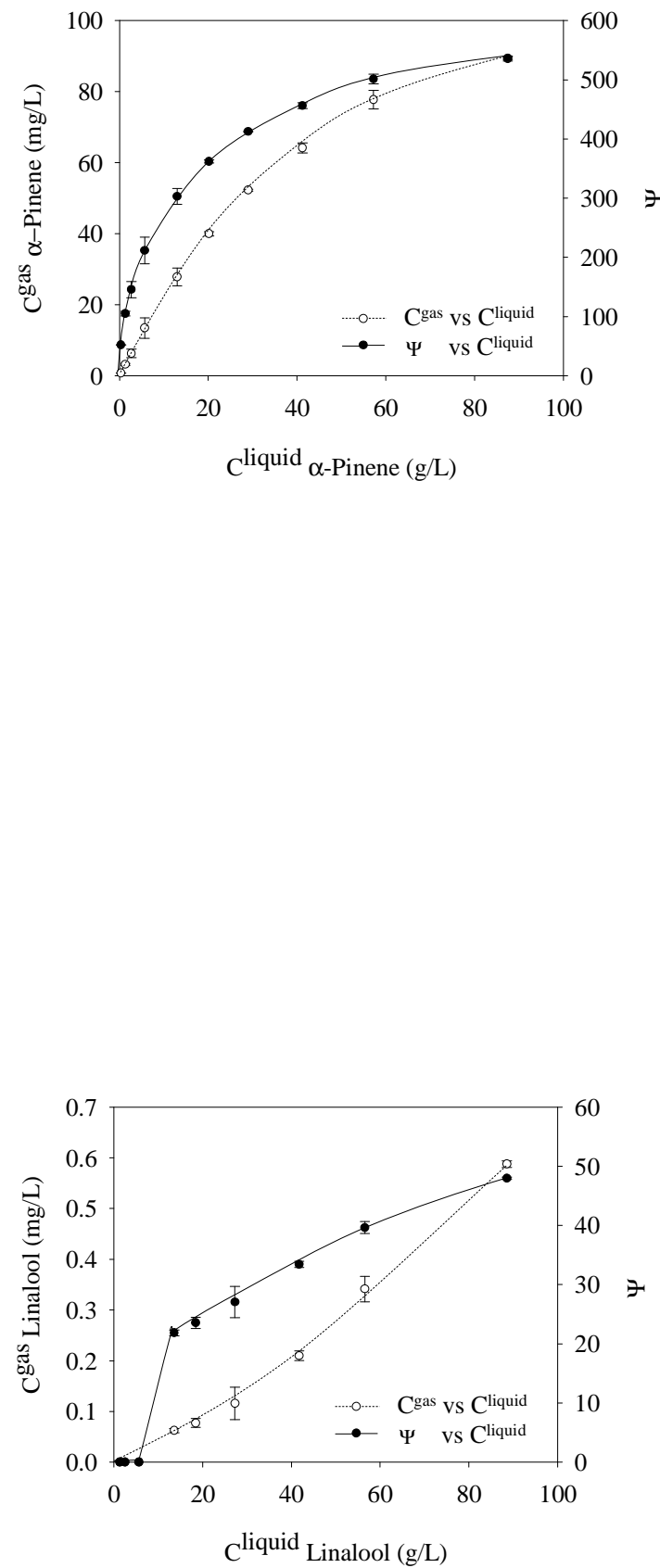
Figure S8. Gas phase concentrations and odor intensities (using the Stevens' power law as odor intensity model) as a function of their liquid phase concentrations for the ternary fragrance mixtures a) limonene $+\alpha$-pinene + linalyl acetate and b) limonene $+p$ cymene + linalool diluted in DPG. 
a) Limonene $+\alpha$-Pinene $+p$-Cymene + Linalyl acetate
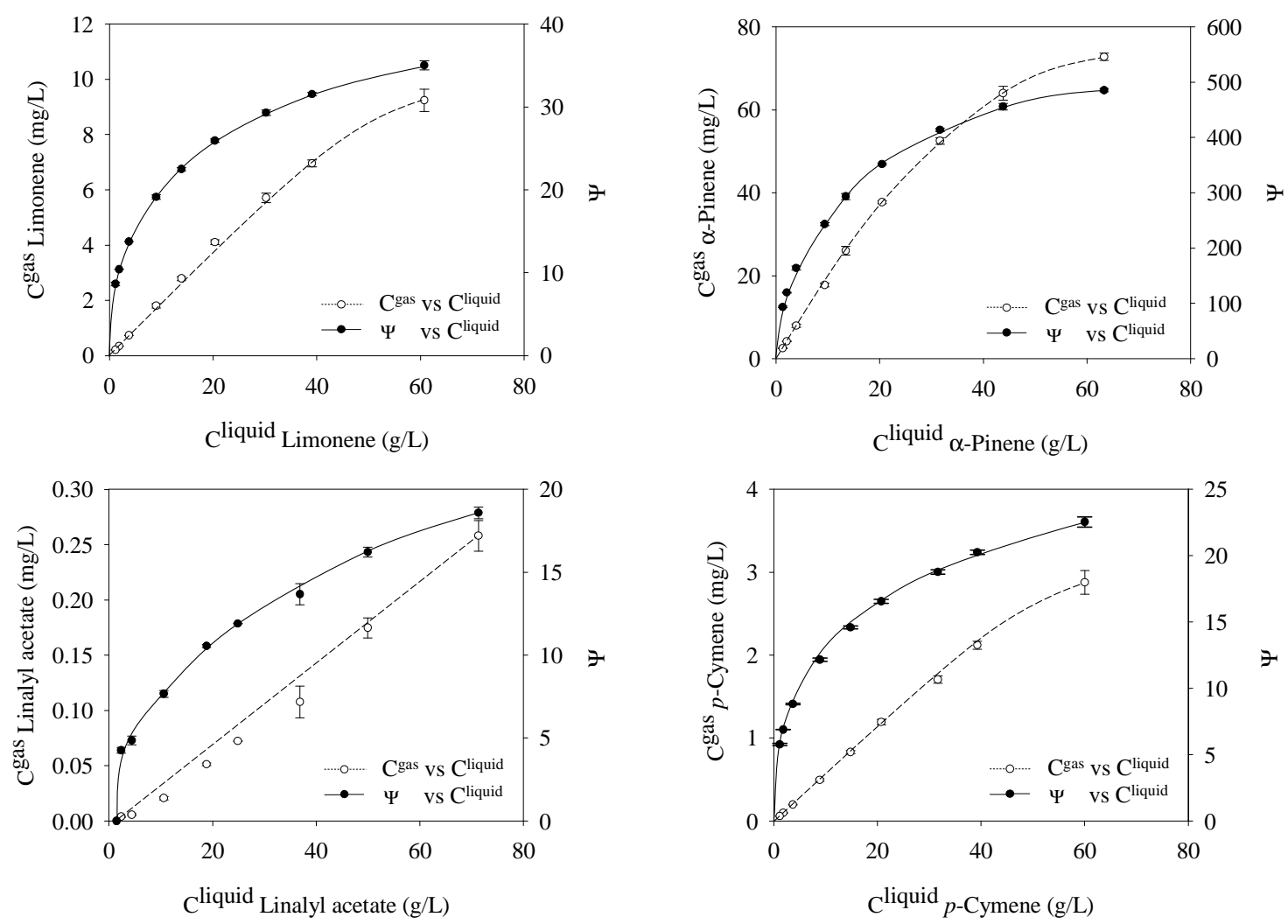

b) Limonene $+\alpha$-Pinene $+p$-Cymene + Linalool
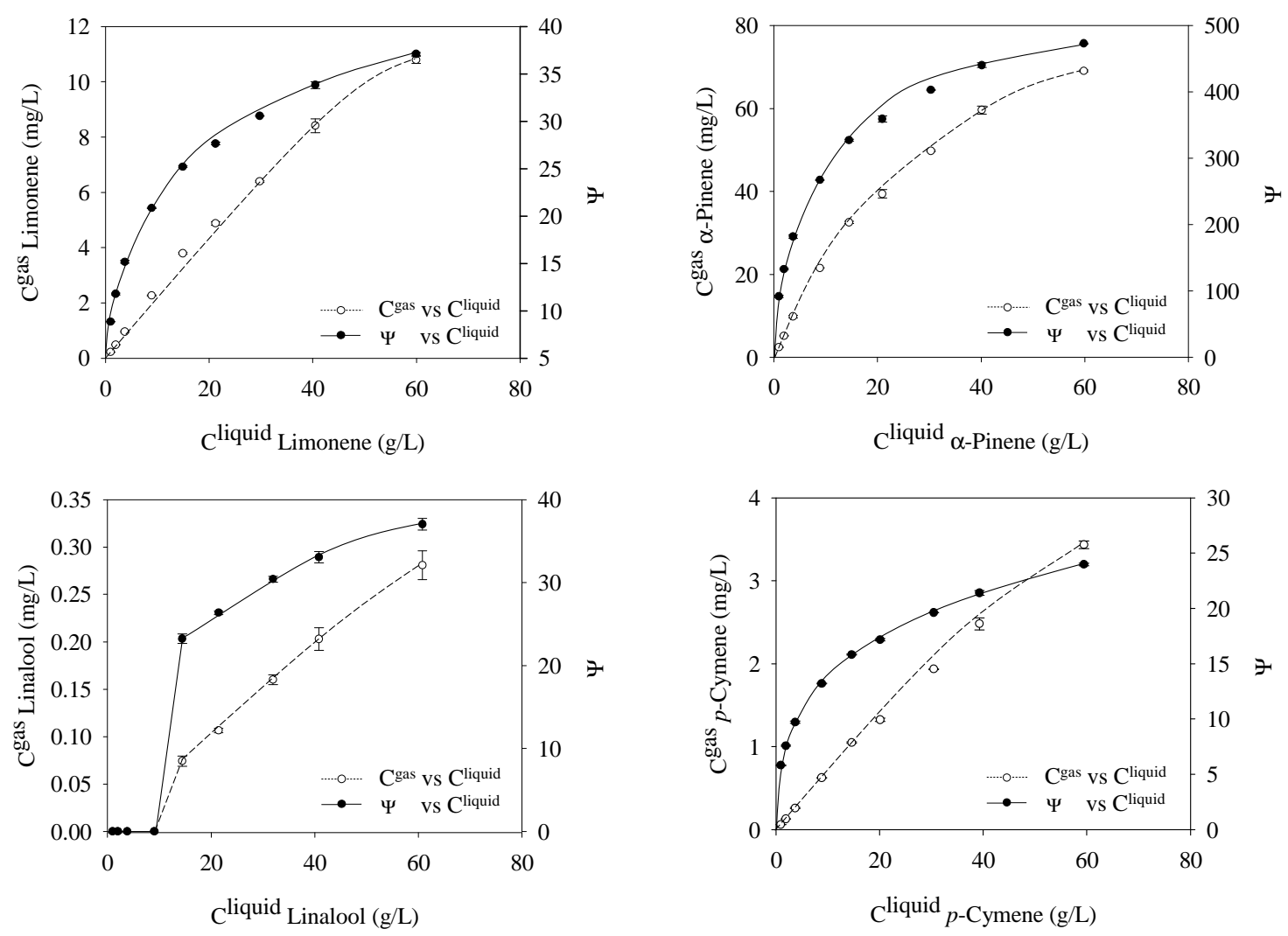
Figure S9. Gas phase concentrations and odor intensities (using the Stevens' power law as odor intensity model) as a function of their liquid phase concentrations for the quaternary fragrance mixtures a) limonene $+\alpha$-pinene $+p$-cymene + linalyl acetate and b) limonene $+\alpha$-pinene $+p$-cymene + linalool diluted in DPG.

Table S1. Studied fragrance mixtures diluted in DPG and total fragrance concentration percentages tested in each fragrance system.

\begin{tabular}{|c|c|}
\hline Components & $\begin{array}{l}\text { Theoretical total fragrance } \\
\text { concentration percentages } \\
(\%) \text { tested in the mixture } \\
(w / w)\end{array}$ \\
\hline $\begin{array}{l}\text { Limonene } \\
\alpha \text {-Pinene } \\
\text { Linalyl acetate } \\
\text { Linalool } \\
\text { Ethyl acetate } \\
\text { Benzyl acetate } \\
p \text {-Cymene }\end{array}$ & $10,5,1,0.1,0.01,0.001$ \\
\hline $\begin{array}{l}\text { Limonene }+\alpha \text {-pinene } \\
\text { Limonene }+ \text { linalyl acetate } \\
\text { Limonene }+ \text { linalool } \\
\text { Limonene }+ \text { ethyl acetate } \\
\text { Limonene }+ \text { benzyl acetate } \\
\text { Limonene }+p \text {-cymene }\end{array}$ & $20,10,5,1,0.1,0.01,0.001$ \\
\hline $\begin{array}{l}\text { Limonene }+\alpha \text {-pinene }+ \text { linalyl acetate } \\
\text { Limonene }+p \text {-cymene }+ \text { linalool } \\
\text { Limonene }+ \text { ethyl acetate }+ \text { benzyl acetate }\end{array}$ & $30,20,15,10,7,5,2,1,0.5$ \\
\hline $\begin{array}{l}\text { Limonene }+\alpha \text {-pinene }+p \text {-cymene }+ \text { linalyl acetate } \\
\text { Limonene }+\alpha \text {-pinene }+p \text {-cymene }+ \text { linalool } \\
\text { Limonene }+p \text {-cymene }+ \text { ethyl acetate }+ \text { benzyl acetate }\end{array}$ & $30,20,15,10,7,5,2,1,0.5$ \\
\hline
\end{tabular}


Table S2. UNIFAC groups and subgroups, the number of groups in molecule $i\left(v_{j}^{(i)}\right)$, the parameters of molecular van der Waals volumes $\left(R_{j}^{(i)}\right)$ and surface area $\left(Q_{j}^{(i)}\right)$.

\begin{tabular}{|c|c|c|c|c|c|c|}
\hline Molecule (i) & Subgroup $(j)$ & $\begin{array}{c}\text { Group } \\
\text { Number }\end{array}$ & $\begin{array}{c}\text { Subgroup } \\
\text { Number }\end{array}$ & $v_{j}^{(i)}$ & $R_{j}^{(i)}$ & $Q_{j}^{(i)}$ \\
\hline \multirow[t]{5}{*}{$\overline{\mathrm{DPG}}$} & $\mathrm{CH}_{3}$ & 1 & 1 & 2 & 0.901 & 0.848 \\
\hline & $\mathrm{CH}_{2}$ & 1 & 2 & 2 & 0.674 & 0.540 \\
\hline & $\mathrm{CH}$ & 1 & 3 & 1 & 0.447 & 0.228 \\
\hline & $\mathrm{OH}$ & 5 & 15 & 2 & 1.000 & 1.200 \\
\hline & $\mathrm{CHO}$ & 13 & 27 & 1 & 0.6908 & 0.468 \\
\hline \multirow[t]{5}{*}{ Limonene } & $\mathrm{CH}_{3}$ & 1 & 1 & 2 & 0.901 & 0.848 \\
\hline & $\mathrm{CH}_{2}$ & 1 & 2 & 3 & 0.674 & 0.540 \\
\hline & $\mathrm{CH}$ & 1 & 3 & 1 & 0.447 & 0.228 \\
\hline & $\mathrm{CH}=\mathrm{C}$ & 2 & 8 & 1 & 0.889 & 0.676 \\
\hline & $\mathrm{CH}_{2}=\mathrm{C}$ & 2 & 7 & 1 & 1.117 & 0.988 \\
\hline \multirow[t]{5}{*}{$\alpha$-Pinene } & $\mathrm{CH}_{3}$ & 1 & 1 & 3 & 0.901 & 0.848 \\
\hline & $\mathrm{CH}_{2}$ & 1 & 2 & 2 & 0.674 & 0.540 \\
\hline & $\mathrm{CH}$ & 1 & 3 & 2 & 0.447 & 0.228 \\
\hline & $\mathrm{CH}=\mathrm{C}$ & 2 & 8 & 1 & 0.889 & 0.676 \\
\hline & $\mathrm{C}$ & 1 & 4 & 1 & 0.220 & 0.000 \\
\hline \multirow[t]{4}{*}{$p$-Cymene } & $\mathrm{ACH}$ & 3 & 10 & 4 & 0.531 & 0.400 \\
\hline & $\mathrm{ACCH}_{3}$ & 4 & 12 & 1 & 1.266 & 0.968 \\
\hline & $\mathrm{ACCH}$ & 4 & 14 & 1 & 0.812 & 0.348 \\
\hline & $\mathrm{CH}_{3}$ & 1 & 1 & 2 & 0.901 & 0.848 \\
\hline \multirow[t]{6}{*}{ Linalool } & $\mathrm{CH}_{3}$ & 1 & 1 & 3 & 0.901 & 0.848 \\
\hline & $\mathrm{CH}_{2}$ & 1 & 2 & 2 & 0.674 & 0.540 \\
\hline & $\mathrm{C}$ & 1 & 4 & 1 & 0.220 & 0.000 \\
\hline & $\mathrm{CH}=\mathrm{C}$ & 2 & 8 & 1 & 0.889 & 0.676 \\
\hline & $\mathrm{CH}_{2}=\mathrm{CH}$ & 2 & 5 & 1 & 1.345 & 1.176 \\
\hline & $\mathrm{OH}^{-}$ & 5 & 15 & 1 & 1.000 & 1.200 \\
\hline \multirow[t]{6}{*}{ Linalyl acetate } & $\mathrm{CH}_{3}$ & 1 & 1 & 3 & 0.901 & 0.848 \\
\hline & $\mathrm{CH}_{2}$ & 1 & 2 & 2 & 0.674 & 0.540 \\
\hline & $\mathrm{C}$ & 1 & 4 & 1 & 0.220 & 0.000 \\
\hline & $\mathrm{CH}=\mathrm{C}$ & 2 & 8 & 1 & 0.889 & 0.676 \\
\hline & $\mathrm{CH}_{2}=\mathrm{CH}$ & 2 & 5 & 1 & 1.345 & 1.176 \\
\hline & $\mathrm{CH}_{3} \mathrm{COO}$ & 11 & 22 & 1 & 1.903 & 1.728 \\
\hline \multirow[t]{3}{*}{ Ethyl acetate } & $\mathrm{CH}_{3}$ & 1 & 1 & 1 & 0.901 & 0.848 \\
\hline & $\mathrm{CH}_{2}$ & 1 & 2 & 1 & 0.674 & 0.540 \\
\hline & $\mathrm{CH}_{3} \mathrm{COO}$ & 11 & 22 & 1 & 1.903 & 1.728 \\
\hline \multirow[t]{3}{*}{ Benzyl acetate } & $\mathrm{ACH}$ & 3 & 10 & 5 & 0.531 & 0.400 \\
\hline & $\mathrm{ACCH}_{2}$ & 4 & 13 & 1 & 1.040 & 0.660 \\
\hline & $\mathrm{CH}_{3} \mathrm{COO}$ & 11 & 22 & 1 & 1.903 & 1.728 \\
\hline
\end{tabular}


Table S3. Theoretical Henry's law constant $\left(H^{\text {Theor }}\right)$ for the single fragrance mixtures diluted in DPG.

\begin{tabular}{lc}
\hline $\begin{array}{l}\text { Single fragrance } \\
\text { mixtures in DPG }\end{array}$ & $\boldsymbol{H}^{\text {Theor }}$ \\
\hline Limonene & $(1.89 \pm 0.24) \times 10^{-4}$ \\
\hline$\alpha$-Pinene & $(4.26 \pm 0.22) \times 10^{-4}$ \\
\hline Linalyl acetate & $(1.29 \pm 0.18) \times 10^{-5}$ \\
\hline Linalool & $(3.64 \pm 0.23) \times 10^{-6}$ \\
\hline Ethyl acetate & $(1.37 \pm 0.04) \times 10^{-3}$ \\
\hline Benzyl acetate & $(8.22 \pm 0.46) \times 10^{-6}$ \\
\hline$p$-Cymene & $(9.83 \pm 0.38) \times 10^{-5}$ \\
\hline
\end{tabular}

Table S4. Experimental Henry's law constant $(\mathrm{H})$ together with the coefficient of determination $\left(\mathrm{r}^{2}\right)$, F value, maximum liquid concentrations $\left(\mathrm{C}^{\text {liquid,max }}, \mathrm{g} / \mathrm{L}\right)$, and relative deviation of $\mathrm{H}\left(\delta_{\mathrm{H}}, \%\right)$ between experimental and theoretical $\mathrm{H}$ for single fragrance mixtures diluted in DPG.

\begin{tabular}{lcclcc}
\hline $\begin{array}{l}\text { Single fragrance } \\
\text { mixtures in DPG }\end{array}$ & \multicolumn{1}{c}{$\mathbf{H}^{\mathbf{a}}$} & $\mathbf{r}^{\mathbf{2}}$ & $\mathbf{F}$ value & $\mathbf{C}^{\text {liquid,max }}(\mathbf{g} / \mathbf{L})^{\mathbf{b}}$ & $\boldsymbol{\delta}_{\mathbf{H}}(\%)$ \\
\hline Limonene & $(2.344 \pm 0.001) \times 10^{-4}$ & $9.996 \times 10^{-1}$ & 12615 & $87.50 \pm 0.02$ & 19.3 \\
\hline$\alpha$-Pinene & $(1.497 \pm 0.093) \times 10^{-3}$ & $9.993 \times 10^{-1}$ & 2812 & $38.93 \pm 0.03$ & 71.5 \\
\hline Linalyl acetate & $(3.542 \pm 0.002) \times 10^{-6}$ & $9.996 \times 10^{-1}$ & 13023 & $97.25 \pm 0.02$ & 264.0 \\
\hline Linalool & $(6.594 \pm 0.238) \times 10^{-6}$ & $9.993 \times 10^{-1}$ & 9355 & $88.28 \pm 0.02$ & 44.8 \\
\hline Ethyl acetate & $(1.739 \pm 0.011) \times 10^{-3}$ & $9.999 \times 10^{-1}$ & 285596 & $49.69 \pm 0.04$ & 21.4 \\
\hline Benzyl acetate & $(5.277 \pm 0.146) \times 10^{-6}$ & $9.999 \times 10^{-1}$ & 21786 & $55.77 \pm 0.05$ & 55.8 \\
\hline$p$-Cymene & $(4.967 \pm 0.013) \times 10^{-5}$ & $9.998 \times 10^{-1}$ & 22571 & $37.69 \pm 0.03$ & 97.8 \\
\hline
\end{tabular}

${ }^{\text {a}}$ Values are expressed as mean \pm standard deviation $(\mathrm{n}=2)$.

b Maximum liquid concentration of each fragrance component where Henry's law is valid. 
Table S5. Experimental Henry's constant $(\mathrm{H})$ together with the coefficient of determination $\left(\mathrm{r}^{2}\right)$, F value, maximum liquid concentrations ( $\left.\mathrm{C}^{\text {liquid,max }}, \mathrm{g} / \mathrm{L}\right)$, and relative deviation of $\mathrm{H}\left(\delta_{\mathrm{H}}, \%\right)$ between experimental and theoretical $\mathrm{H}$ for the studied mixtures.

\begin{tabular}{|c|c|c|c|c|c|}
\hline & $\mathbf{H}$ & $\mathbf{r}^{2}$ & F value & $\mathrm{C}^{\text {liquid,max }}(\mathrm{g} / \mathrm{L})^{\mathrm{b}}$ & $\delta_{H}(\%)$ \\
\hline \multicolumn{6}{|c|}{ Binary fragrance mixtures } \\
\hline Limonene & $(1.914 \pm 0.004) \times 10^{-4}$ & $9.973 \times 10^{-1}$ & 1519 & $43.77 \pm 0.04$ & 7.7 \\
\hline$\alpha$-Pinene & $(1.812 \pm 0.009) \times 10^{-3}$ & $9.987 \times 10^{-1}$ & 2528 & $21.06 \pm 0.07$ & 78.4 \\
\hline Limonene & $(1.640 \pm 0.042) \times 10^{-4}$ & $9.998 \times 10^{-1}$ & 50314 & $43.71 \pm 0.03$ & 8.2 \\
\hline Linalyl acetate & $(3.193 \pm 0.156) \times 10^{-6}$ & $9.998 \times 10^{-1}$ & 2560 & $46.11 \pm 0.04$ & 271.0 \\
\hline Limonene & $(2.033 \pm 0.010) \times 10^{-4}$ & $9.915 \times 10^{-1}$ & 357 & $41.89 \pm 0.03$ & 12.2 \\
\hline Linalool & $(6.918 \pm 0.032) \times 10^{-6}$ & $9.974 \times 10^{-1}$ & 1142 & $87.74 \pm 0.02$ & 53.1 \\
\hline Limonene & $(2.541 \pm 0.057) \times 10^{-4}$ & $9.954 \times 10^{-1}$ & 387 & $21.71 \pm 0.07$ & 28.0 \\
\hline Benzyl acetate & $(5.385 \pm 0.045) \times 10^{-6}$ & $9.985 \times 10^{-1}$ & 1250 & $28.3 \pm 0.1$ & 41.2 \\
\hline Limonene & $(1.887 \pm 0.025) \times 10^{-4}$ & $9.987 \times 10^{-1}$ & 2255 & $44.02 \pm 0.03$ & 3.3 \\
\hline$p$-Cymene & $(5.299 \pm 0.099) \times 10^{-5}$ & $9.995 \times 10^{-1}$ & 7406 & $44.84 \pm 0.03$ & 66.2 \\
\hline Limonene & $(2.831 \pm 0.018) \times 10^{-4}$ & $9.968 \times 10^{-1}$ & 628 & $22.9 \pm 0.1$ & 35.9 \\
\hline Ethyl acetate & $(2.230 \pm 0.131) \times 10^{-3}$ & $9.979 \times 10^{-1}$ & 3211 & $23.32 \pm 0.07$ & 43.8 \\
\hline \multicolumn{6}{|c|}{ Ternary fragrance mixtures } \\
\hline Limonene & $(1.993 \pm 0.097) \times 10^{-4}$ & $9.942 \times 10^{-1}$ & 900 & $42.19 \pm 0.06$ & 11.8 \\
\hline$\alpha$-Pinene & $(1.989 \pm 0.083) \times 10^{-3}$ & $9.935 \times 10^{-1}$ & 488 & $20.2 \pm 0.2$ & 79.5 \\
\hline Linalyl acetate & $(2.999 \pm 0.343) \times 10^{-6}$ & $9.924 \times 10^{-1}$ & 566 & $20.9 \pm 0.1$ & 320.1 \\
\hline Limonene & $(1.387 \pm 0.008) \times 10^{-4}$ & $9.826 \times 10^{-1}$ & 338 & $54.05 \pm 0.07$ & 28.1 \\
\hline Ethyl acetate & $(2.259 \pm 0.001) \times 10^{-3}$ & $9.995 \times 10^{-1}$ & 8358 & $19.6 \pm 0.1$ & 40.6 \\
\hline Benzyl acetate & $(5.527 \pm 0.042) \times 10^{-6}$ & $9.978 \times 10^{-1}$ & 3243 & $56.55 \pm 0.04$ & 31.9 \\
\hline Limonene & $(1.978 \pm 0.006) \times 10^{-4}$ & $9.928 \times 10^{-1}$ & 1040 & $43.13 \pm 0.05$ & 10.2 \\
\hline$p$-Cymene & $(5.851 \pm 0.019) \times 10^{-5}$ & $9.948 \times 10^{-1}$ & 953 & $44.83 \pm 0.05$ & 53.0 \\
\hline Linalool & $(7.021 \pm 0.003) \times 10^{-6}$ & $9.088 \times 10^{-1}$ & 23 & $88.59 \pm 0.02$ & 54.9 \\
\hline \multicolumn{6}{|c|}{ Quaternary fragrance mixtures } \\
\hline Limonene & $(1.922 \pm 0.037) \times 10^{-4}$ & $9.965 \times 10^{-1}$ & 2660 & $30.22 \pm 0.07$ & 4.3 \\
\hline$\alpha$-Pinene & $(1.844 \pm 0.006) \times 10^{-3}$ & $9.968 \times 10^{-1}$ & 2278 & $20.4 \pm 0.1$ & 78.3 \\
\hline$p$-Cymene & $(5.422 \pm 0.127) \times 10^{-5}$ & $9.981 \times 10^{-1}$ & 8278 & $39.31 \pm 0.07$ & 58.7 \\
\hline Linalyl acetate & $(3.685 \pm 0.047) \times 10^{-6}$ & $9.633 \times 10^{-1}$ & 201 & $71.30 \pm 0.03$ & 184.0 \\
\hline Limonene & $(1.416 \pm 0.011) \times 10^{-4}$ & $9.912 \times 10^{-1}$ & 461 & $20.8 \pm 0.1$ & 31.6 \\
\hline$p$-Cymene & $(4.135 \pm 0.115) \times 10^{-5}$ & $9.687 \times 10^{-1}$ & 195 & $40.40 \pm 0.05$ & 110.6 \\
\hline Ethyl acetate & $(2.321 \pm 0.006) \times 10^{-3}$ & $9.990 \times 10^{-1}$ & 4527 & $20.9 \pm 0.1$ & 42.5 \\
\hline Benzyl acetate & $(5.641 \pm 0.080) \times 10^{-6}$ & $9.994 \times 10^{-1}$ & 13448 & $39.5 \pm 0.1$ & 35.0 \\
\hline Limonene & $(2.256 \pm 0.154) \times 10^{-4}$ & $9.933 \times 10^{-1}$ & 517 & $21.2 \pm 0.1$ & 18.2 \\
\hline$\alpha$-Pinene & $(1.899 \pm 0.030) \times 10^{-3}$ & $9.499 \times 10^{-1}$ & 77 & $20.9 \pm 0.1$ & 78.9 \\
\hline$p$-Cymene & $(6.289 \pm 0.132) \times 10^{-5}$ & $9.950 \times 10^{-1}$ & 1289 & $39.3 \pm 0.1$ & 38.1 \\
\hline Linalool & $(5.100 \pm 0.147) \times 10^{-6}$ & $9.342 \times 10^{-1}$ & 56 & $60.74 \pm 0.03$ & 37.5 \\
\hline
\end{tabular}


${ }^{a}$ Values are expressed as mean \pm standard deviation $(\mathrm{n}=2)$.

$p$-value $<0.05$

${ }^{\mathrm{b}}$ Maximum liquid concentration of each fragrance component where Henry's law is valid. 
Table S6. Experimental molar compositions of the liquid $(x)$ phase and predicted molar composition of the vapor $(y)$ phase and activity coefficient $(\gamma)$ using the UNIFAC method for the each component in the mixtures.

\begin{tabular}{llllll}
\hline DPG & & \multicolumn{4}{l}{ Limonene } \\
\hline$x$ & $y$ & $\gamma$ & $x$ & $y$ & $\gamma$ \\
\hline $9.14 \times 10^{-1}$ & $1.41 \times 10^{-2}$ & 1.02 & $8.63 \times 10^{-2}$ & $9.86 \times 10^{-1}$ & 13.10 \\
$9.56 \times 10^{-1}$ & $2.40 \times 10^{-2}$ & 1.00 & $4.38 \times 10^{-2}$ & $9.76 \times 10^{-1}$ & 15.57 \\
$9.92 \times 10^{-1}$ & $1.02 \times 10^{-1}$ & 1.00 & $8.41 \times 10^{-3}$ & $8.98 \times 10^{-1}$ & 18.03 \\
$9.99 \times 10^{-1}$ & $5.40 \times 10^{-1}$ & 1.00 & $7.98 \times 10^{-4}$ & $4.60 \times 10^{-1}$ & 18.61 \\
$1.00 \times 10^{0}$ & $9.25 \times 10^{-1}$ & 1.00 & $7.57 \times 10^{-5}$ & $7.50 \times 10^{-2}$ & 18.67 \\
$1.00 \times 10^{0}$ & $9.92 \times 10^{-1}$ & 1.00 & $7.29 \times 10^{-6}$ & $7.75 \times 10^{-3}$ & 18.68 \\
& & & & & \\
\hline DPG & & & $\alpha-$ Pinene & & \\
\hline$x$ & $y$ & $\gamma$ & $x$ & $y$ & $\gamma$ \\
\hline $9.10 \times 10^{-1}$ & $6.05 \times 10^{-3}$ & 1.02 & $8.97 \times 10^{-2}$ & $9.94 \times 10^{-1}$ & 11.80 \\
$9.62 \times 10^{-1}$ & $1.24 \times 10^{-2}$ & 1.00 & $3.79 \times 10^{-2}$ & $9.88 \times 10^{-1}$ & 14.07 \\
$9.91 \times 10^{-1}$ & $4.57 \times 10^{-2}$ & 1.00 & $9.30 \times 10^{-3}$ & $9.54 \times 10^{-1}$ & 15.52 \\
$9.99 \times 10^{-1}$ & $3.77 \times 10^{-1}$ & 1.00 & $7.22 \times 10^{-4}$ & $6.23 \times 10^{-1}$ & 15.98 \\
$1.00 \times 10^{0}$ & $8.56 \times 10^{-1}$ & 1.00 & $7.35 \times 10^{-5}$ & $1.44 \times 10^{-1}$ & 16.02 \\
$1.00 \times 10^{0}$ & $9.82 \times 10^{-1}$ & 1.00 & $7.77 \times 10^{-6}$ & $1.75 \times 10^{-2}$ & 16.02
\end{tabular}

\begin{tabular}{llllll}
\hline DPG & & \multicolumn{4}{l}{ Linalyl acetate } \\
\hline$x$ & $y$ & $\gamma$ & $x$ & $y$ & $\gamma$ \\
\hline $9.32 \times 10^{-1}$ & $2.20 \times 10^{-1}$ & 1.01 & $6.82 \times 10^{-2}$ & $7.80 \times 10^{-1}$ & 11.90 \\
$9.94 \times 10^{-1}$ & $6.96 \times 10^{-1}$ & 1.00 & $6.20 \times 10^{-3}$ & $3.04 \times 10^{-1}$ & 16.91 \\
$9.99 \times 10^{-1}$ & $9.60 \times 10^{-1}$ & 1.00 & $5.77 \times 10^{-4}$ & $4.01 \times 10^{-2}$ & 17.49 \\
$1.00 \times 10^{0}$ & $9.95 \times 10^{-1}$ & 1.00 & $6.79 \times 10^{-5}$ & $4.90 \times 10^{-3}$ & 17.54 \\
$1.00 \times 10^{0}$ & $1.00 \times 10^{0}$ & 1.00 & $6.79 \times 10^{-6}$ & $4.93 \times 10^{-4}$ & 17.55
\end{tabular}

\begin{tabular}{|c|c|c|c|c|c|}
\hline \multicolumn{3}{|l|}{ DPG } & \multicolumn{3}{|l|}{ Linalool } \\
\hline$x$ & $y$ & $\gamma$ & $x$ & $y$ & $\gamma$ \\
\hline $9.22 \times 10^{-1}$ & $4.22 \times 10^{-1}$ & 1.01 & $7.75 \times 10^{-2}$ & $5.78 \times 10^{-1}$ & 2.66 \\
\hline $9.63 \times 10^{-1}$ & $5.91 \times 10^{-1}$ & 1.00 & $3.70 \times 10^{-2}$ & $4.09 \times 10^{-1}$ & 2.93 \\
\hline $9.93 \times 10^{-1}$ & $8.75 \times 10^{-1}$ & 1.00 & $7.31 \times 10^{-3}$ & $1.25 \times 10^{-1}$ & 3.15 \\
\hline $9.99 \times 10^{-1}$ & $9.86 \times 10^{-1}$ & 1.00 & $6.99 \times 10^{-4}$ & $1.36 \times 10^{-2}$ & 3.20 \\
\hline $1.00 \times 10^{0}$ & $9.99 \times 10^{-1}$ & 1.00 & $7.35 \times 10^{-5}$ & $1.45 \times 10^{-3}$ & 3.21 \\
\hline $1.00 \times 10^{0}$ & $1.00 \times 10^{0}$ & 1.00 & $8.47 \times 10^{-6}$ & $1.68 \times 10^{-4}$ & 3.21 \\
\hline \multicolumn{3}{|l|}{ DPG } & \multicolumn{3}{|c|}{ Benzyl acetate } \\
\hline$x$ & $y$ & $\gamma$ & $x$ & $y$ & $\gamma$ \\
\hline $9.03 \times 10^{-1}$ & $2.18 \times 10^{-1}$ & 1.01 & $9.74 \times 10^{-2}$ & $7.82 \times 10^{-1}$ & 5.509 \\
\hline $9.51 \times 10^{-1}$ & $3.35 \times 10^{-1}$ & 1.00 & $4.91 \times 10^{-2}$ & $6.65 \times 10^{-1}$ & 6.299 \\
\hline $9.92 \times 10^{-1}$ & $7.35 \times 10^{-1}$ & 1.00 & $8.27 \times 10^{-3}$ & $2.65 \times 10^{-1}$ & 7.064 \\
\hline $9.99 \times 10^{-1}$ & $9.62 \times 10^{-1}$ & 1.00 & $8.90 \times 10^{-4}$ & $3.78 \times 10^{-2}$ & 7.212 \\
\hline $1.00 \times 10^{0}$ & $9.96 \times 10^{-1}$ & 1.00 & $8.48 \times 10^{-5}$ & $3.73 \times 10^{-3}$ & 7.229 \\
\hline $1.00 \mathrm{E} \times 10^{0}$ & $1.00 \times 10^{0}$ & 1.00 & $8.35 \times 10^{-6}$ & $3.69 \times 10^{-4}$ & 7.230 \\
\hline \multicolumn{3}{|l|}{ DPG } & \multicolumn{3}{|c|}{ Ethyl acetate } \\
\hline$x$ & $y$ & $\gamma$ & $x$ & $y$ & $\gamma$ \\
\hline $8.66 \times 10^{-1}$ & $9.76 \times 10^{-4}$ & 1.01 & $1.34 \times 10^{-1}$ & $9.99 \times 10^{-1}$ & 1.92 \\
\hline $9.27 \times 10^{-1}$ & $1.83 \times 10^{-3}$ & 1.00 & $7.29 \times 10^{-2}$ & $9.98 \times 10^{-1}$ & 2.00 \\
\hline $9.85 \times 10^{-1}$ & $9.09 \times 10^{-3}$ & 1.00 & $1.50 \times 10^{-2}$ & $9.91 \times 10^{-1}$ & 2.07 \\
\hline $9.99 \times 10^{-1}$ & $9.36 \times 10^{-2}$ & 1.00 & $1.34 \times 10^{-3}$ & $9.06 \times 10^{-1}$ & 2.08 \\
\hline $1.00 \times 10^{0}$ & $4.98 \times 10^{-1}$ & 1.00 & $1.39 \times 10^{-4}$ & $5.02 \times 10^{-1}$ & 2.08 \\
\hline $1.00 \times 10^{0}$ & $8.99 \times 10^{-1}$ & 1.00 & $1.55 \times 10^{-5}$ & $1.01 \times 10^{-1}$ & 2.09 \\
\hline \multicolumn{3}{|l|}{ DPG } & \multicolumn{3}{|l|}{$p$-Cymene } \\
\hline$x$ & $y$ & $\gamma$ & $x$ & $y$ & $\gamma$ \\
\hline
\end{tabular}




\begin{tabular}{llllll}
\hline $9.03 \times 10^{-1}$ & $2.28 \times 10^{-2}$ & 1.01 & $9.67 \times 10^{-2}$ & $9.77 \times 10^{-1}$ & 7.27 \\
$9.63 \times 10^{-1}$ & $5.17 \times 10^{-2}$ & 1.00 & $3.73 \times 10^{-2}$ & $9.48 \times 10^{-1}$ & 8.50 \\
$9.92 \times 10^{-1}$ & $1.96 \times 10^{-1}$ & 1.00 & $7.92 \times 10^{-3}$ & $8.04 \times 10^{-1}$ & 9.19 \\
$9.99 \times 10^{-1}$ & $7.26 \times 10^{-1}$ & 1.00 & $7.20 \times 10^{-4}$ & $2.74 \times 10^{-1}$ & 9.36 \\
$1.00 \times 10^{0}$ & $9.64 \times 10^{-1}$ & 1.00 & $7.06 \times 10^{-5}$ & $3.57 \times 10^{-2}$ & 9.38 \\
$1.00 \times 10^{0}$ & $9.96 \times 10^{-1}$ & 1.00 & $8.01 \times 10^{-6}$ & $4.18 \times 10^{-3}$ & 9.38 \\
\hline
\end{tabular}


Table S7. Experimental molar compositions of the liquid $(x)$ phase and predicted molar composition of the vapor $(y)$ phase and activity coefficient $(\gamma)$ using the UNIFAC model for the binary fragrance mixtures.

\begin{tabular}{|c|c|c|c|c|c|c|c|c|}
\hline \multicolumn{3}{|l|}{ DPG } & \multicolumn{3}{|l|}{ Limonene } & \multicolumn{3}{|l|}{$\alpha$-Pinene } \\
\hline$x$ & $y$ & $\gamma$ & $x$ & $y$ & $\gamma$ & $x$ & $y$ & $\gamma$ \\
\hline $8.25 \times 10^{-1}$ & $5.53 \times 10^{-3}$ & 1.06 & $8.67 \times 10^{-2}$ & $2.99 \times 10^{-1}$ & 9.56 & $8.82 \times 10^{-2}$ & $6.95 \times 10^{-1}$ & 8.73 \\
\hline $9.14 \times 10^{-1}$ & $8.71 \times 10^{-3}$ & 1.02 & $4.31 \times 10^{-2}$ & $3.10 \times 10^{-1}$ & 13.36 & $4.27 \times 10^{-2}$ & $6.81 \times 10^{-1}$ & 11.83 \\
\hline $9.59 \times 10^{-1}$ & $1.59 \times 10^{-2}$ & 1.00 & $2.04 \times 10^{-2}$ & $3.08 \times 10^{-1}$ & 15.90 & $2.05 \times 10^{-2}$ & $6.76 \times 10^{-1}$ & 13.85 \\
\hline $9.92 \times 10^{-1}$ & $6.90 \times 10^{-2}$ & 1.00 & $4.09 \times 10^{-3}$ & $2.95 \times 10^{-1}$ & 18.08 & $4.09 \times 10^{-3}$ & $6.35 \times 10^{-1}$ & 15.56 \\
\hline $9.99 \times 10^{-1}$ & $4.18 \times 10^{-1}$ & 1.00 & $4.15 \times 10^{-4}$ & $1.85 \times 10^{-1}$ & 18.62 & $4.15 \times 10^{-4}$ & $3.97 \times 10^{-1}$ & 15.97 \\
\hline $1.00 \times 10^{0}$ & $8.89 \times 10^{-1}$ & 1.00 & $3.69 \times 10^{-5}$ & $3.52 \times 10^{-2}$ & 18.67 & $3.69 \times 10^{-5}$ & $7.55 \times 10^{-2}$ & 16.02 \\
\hline $1.00 \times 10^{0}$ & $9.87 \times 10^{-1}$ & 1.00 & $4.05 \times 10^{-6}$ & $4.28 \times 10^{-3}$ & 18.68 & $4.05 \times 10^{-6}$ & $9.18 \times 10^{-3}$ & 16.02 \\
\hline \multicolumn{3}{|l|}{ DPG } & \multicolumn{3}{|l|}{ Limonene } & \multicolumn{3}{|c|}{ Linalyl acetate } \\
\hline$x$ & $y$ & $\gamma$ & $x$ & $y$ & $\gamma$ & $x$ & $y$ & $\gamma$ \\
\hline $8.43 \times 10^{-1}$ & $1.67 \times 10^{-2}$ & 1.06 & $9.09 \times 10^{-2}$ & $9.42 \times 10^{-1}$ & 9.68 & $6.65 \times 10^{-2}$ & $4.15 \times 10^{-2}$ & 8.10 \\
\hline $9.25 \times 10^{-1}$ & $2.59 \times 10^{-2}$ & 1.01 & $4.36 \times 10^{-2}$ & $9.31 \times 10^{-1}$ & 13.48 & $3.19 \times 10^{-2}$ & $4.34 \times 10^{-2}$ & 11.93 \\
\hline $9.62 \times 10^{-1}$ & $4.46 \times 10^{-2}$ & 1.00 & $2.18 \times 10^{-2}$ & $9.11 \times 10^{-1}$ & 15.80 & $1.63 \times 10^{-2}$ & $4.48 \times 10^{-2}$ & 14.39 \\
\hline $9.93 \times 10^{-1}$ & $1.72 \times 10^{-1}$ & 1.00 & $4.39 \times 10^{-3}$ & $7.91 \times 10^{-1}$ & 18.07 & $3.05 \times 10^{-3}$ & $3.69 \times 10^{-2}$ & 16.87 \\
\hline $9.99 \times 10^{-1}$ & $6.93 \times 10^{-1}$ & 1.00 & $3.96 \times 10^{-4}$ & $2.93 \times 10^{-1}$ & 18.62 & $2.75 \times 10^{-4}$ & $1.38 \times 10^{-2}$ & 17.49 \\
\hline $1.00 \times 10^{0}$ & $9.65 \times 10^{-1}$ & 1.00 & $3.27 \times 10^{-5}$ & $3.37 \times 10^{-2}$ & 18.67 & $2.27 \times 10^{-5}$ & $1.59 \times 10^{-3}$ & 17.55 \\
\hline $1.00 \times 10^{0}$ & $9.96 \times 10^{-1}$ & 1.00 & $3.43 \times 10^{-6}$ & $3.66 \times 10^{-3}$ & 18.68 & $2.38 \times 10^{-6}$ & $1.72 \times 10^{-4}$ & 17.55 \\
\hline \multicolumn{3}{|l|}{ DPG } & \multicolumn{3}{|l|}{ Limonene } & \multicolumn{3}{|l|}{ Linalool } \\
\hline$x$ & $y$ & $\gamma$ & $x$ & $y$ & $\gamma$ & $x$ & $y$ & $\gamma$ \\
\hline $8.34 \times 10^{-1}$ & $1.62 \times 10^{-2}$ & 1.05 & $8.77 \times 10^{-2}$ & $9.65 \times 10^{-1}$ & 10.35 & $7.86 \times 10^{-2}$ & $1.86 \times 10^{-2}$ & 2.07 \\
\hline $9.20 \times 10^{-1}$ & $2.68 \times 10^{-2}$ & 1.01 & $4.15 \times 10^{-2}$ & $9.56 \times 10^{-1}$ & 13.96 & $3.81 \times 10^{-2}$ & $1.74 \times 10^{-2}$ & 2.57 \\
\hline $9.60 \times 10^{-1}$ & $4.66 \times 10^{-2}$ & 1.00 & $2.09 \times 10^{-2}$ & $9.37 \times 10^{-1}$ & 16.12 & $1.87 \times 10^{-2}$ & $1.60 \times 10^{-2}$ & 2.86 \\
\hline $9.92 \times 10^{-1}$ & $1.83 \times 10^{-1}$ & 1.00 & $4.20 \times 10^{-3}$ & $8.04 \times 10^{-1}$ & 18.13 & $3.71 \times 10^{-3}$ & $1.32 \times 10^{-2}$ & 3.14 \\
\hline $9.99 \times 10^{-1}$ & $6.90 \times 10^{-1}$ & 1.00 & $4.14 \times 10^{-4}$ & $3.05 \times 10^{-1}$ & 18.62 & $3.66 \times 10^{-4}$ & $4.99 \times 10^{-3}$ & 3.20 \\
\hline $1.00 \times 10^{0}$ & $9.62 \times 10^{-1}$ & 1.00 & $3.60 \times 10^{-5}$ & $3.71 \times 10^{-2}$ & 18.67 & $3.18 \times 10^{-5}$ & $6.06 \times 10^{-4}$ & 3.21 \\
\hline $1.00 \times 10^{0}$ & $9.96 \times 10^{-1}$ & 1.00 & $4.02 \times 10^{-6}$ & $4.29 \times 10^{-3}$ & 18.68 & $3.55 \times 10^{-6}$ & $7.00 \times 10^{-5}$ & 3.21 \\
\hline \multicolumn{3}{|l|}{ DPG } & \multicolumn{3}{|l|}{ Limonene } & \multicolumn{3}{|c|}{ Benzyl acetate } \\
\hline$x$ & $y$ & $\gamma$ & $x$ & $y$ & $\gamma$ & $x$ & $y$ & $\gamma$ \\
\hline $8.27 \times 10^{-1}$ & $1.45 \times 10^{-2}$ & 1.05 & $9.31 \times 10^{-2}$ & $9.47 \times 10^{-1}$ & 10.66 & $7.95 \times 10^{-2}$ & $3.83 \times 10^{-2}$ & 4.72 \\
\hline
\end{tabular}




\begin{tabular}{|c|c|c|c|c|c|c|c|c|}
\hline $9.06 \times 10^{-1}$ & $2.51 \times 10^{-2}$ & 1.01 & $4.24 \times 10^{-2}$ & $9.26 \times 10^{-1}$ & 13.90 & $5.15 \times 10^{-2}$ & $4.90 \times 10^{-2}$ & 5.68 \\
\hline $9.54 \times 10^{-1}$ & $4.45 \times 10^{-2}$ & 1.00 & $2.11 \times 10^{-2}$ & $9.10 \times 10^{-1}$ & 16.13 & $2.50 \times 10^{-2}$ & $4.57 \times 10^{-2}$ & 6.42 \\
\hline $9.92 \times 10^{-1}$ & $1.76 \times 10^{-1}$ & 1.00 & $4.30 \times 10^{-3}$ & $7.94 \times 10^{-1}$ & 18.17 & $3.90 \times 10^{-3}$ & $2.99 \times 10^{-2}$ & 7.08 \\
\hline $1.00 \times 10^{0}$ & $8.32 \times 10^{-1}$ & 1.00 & $1.82 \times 10^{-4}$ & $1.62 \times 10^{-1}$ & 18.66 & $1.65 \times 10^{-4}$ & $6.08 \times 10^{-3}$ & 7.22 \\
\hline $1.00 \times 10^{0}$ & $9.82 \times 10^{-1}$ & 1.00 & $1.62 \times 10^{-5}$ & $1.70 \times 10^{-2}$ & 18.67 & $1.47 \times 10^{-5}$ & $6.38 \times 10^{-4}$ & 7.23 \\
\hline $1.00 \times 10^{0}$ & $9.98 \times 10^{-1}$ & 1.00 & $1.79 \times 10^{-6}$ & $1.92 \times 10^{-3}$ & 18.68 & $1.63 \times 10^{-6}$ & $7.18 \times 10^{-5}$ & 7.23 \\
\hline DPG & & & Limonene & & & Ethyl aceta & & \\
\hline$x$ & $y$ & $\gamma$ & $x$ & $y$ & $\gamma$ & $x$ & $y$ & $\gamma$ \\
\hline $7.76 \times 10^{-1}$ & $9.25 \times 10^{-4}$ & 1.05 & $8.58 \times 10^{-2}$ & $5.96 \times 10^{-2}$ & 10.62 & $1.38 \times 10^{-1}$ & $9.39 \times 10^{-1}$ & 1.72 \\
\hline $8.89 \times 10^{-1}$ & $1.85 \times 10^{-3}$ & 1.01 & $4.25 \times 10^{-2}$ & $7.15 \times 10^{-2}$ & 14.19 & $6.82 \times 10^{-1}$ & $9.27 \times 10^{-1}$ & 1.90 \\
\hline $9.43 \times 10^{-1}$ & $3.62 \times 10^{-3}$ & 1.00 & $2.20 \times 10^{-2}$ & $7.87 \times 10^{-2}$ & 16.23 & $3.47 \times 10^{-2}$ & $9.18 \times 10^{-1}$ & 1.99 \\
\hline $9.88 \times 10^{-1}$ & $1.68 \times 10^{-2}$ & 1.00 & $4.77 \times 10^{-3}$ & $8.44 \times 10^{-2}$ & 18.13 & $7.37 \times 10^{-3}$ & $8.99 \times 10^{-1}$ & 2.06 \\
\hline $9.99 \times 10^{-1}$ & $1.64 \times 10^{-1}$ & 1.00 & $4.16 \times 10^{-4}$ & $7.30 \times 10^{-2}$ & 18.63 & $6.44 \times 10^{-4}$ & $7.63 \times 10^{-1}$ & 2.08 \\
\hline $1.00 \times 10^{0}$ & $6.75 \times 10^{-1}$ & 1.00 & $3.93 \times 10^{-5}$ & $2.84 \times 10^{-2}$ & 18.67 & $6.07 \times 10^{-5}$ & $2.97 \times 10^{-1}$ & 2.08 \\
\hline $1.00 \times 10^{0}$ & $9.47 \times 10^{-1}$ & 1.00 & $4.55 \times 10^{-6}$ & $4.61 \times 10^{-2}$ & 18.68 & $7.03 \times 10^{-6}$ & $4.82 \times 10^{-2}$ & 2.09 \\
\hline DPG & & & Limonene & & & $p$-Cymene & & \\
\hline$x$ & $y$ & $\gamma$ & $x$ & $y$ & $\gamma$ & $x$ & $y$ & $\gamma$ \\
\hline $8.23 \times 10^{-1}$ & $1.12 \times 10^{-2}$ & 1.06 & $8.67 \times 10^{-2}$ & $6.26 \times 10^{-1}$ & 9.79 & $8.99 \times 10^{-2}$ & $3.63 \times 10^{-1}$ & 5.63 \\
\hline $9.12 \times 10^{-1}$ & $1.76 \times 10^{-2}$ & 1.01 & $4.34 \times 10^{-2}$ & $6.37 \times 10^{-1}$ & 13.45 & $4.48 \times 10^{-2}$ & $3.45 \times 10^{-1}$ & 7.23 \\
\hline $9.60 \times 10^{-1}$ & $3.45 \times 10^{-2}$ & 1.00 & $1.84 \times 10^{-2}$ & $6.09 \times 10^{-1}$ & 16.10 & $2.14 \times 10^{-2}$ & $3.56 \times 10^{-1}$ & 8.34 \\
\hline $9.92 \times 10^{-1}$ & $1.46 \times 10^{-1}$ & 1.00 & $3.73 \times 10^{-3}$ & $5.70 \times 10^{-1}$ & 18.15 & $3.79 \times 10^{-3}$ & $2.85 \times 10^{-1}$ & 9.17 \\
\hline $9.99 \times 10^{-1}$ & $6.55 \times 10^{-1}$ & 1.00 & $3.29 \times 10^{-4}$ & $2.30 \times 10^{-1}$ & 18.63 & $3.34 \times 10^{-4}$ & $1.14 \times 10^{-1}$ & 9.36 \\
\hline $1.00 \times 10^{0}$ & $9.56 \times 10^{-1}$ & 1.00 & $2.88 \times 10^{-5}$ & $2.95 \times 10^{-2}$ & 18.67 & $2.92 \times 10^{-5}$ & $1.46 \times 10^{-2}$ & 9.38 \\
\hline $1.00 \times 10^{0}$ & $9.95 \times 10^{-1}$ & 1.00 & $3.25 \times 10^{-6}$ & $3.46 \times 10^{-3}$ & 18.68 & $3.29 \times 10^{-6}$ & $1.72 \times 10^{-3}$ & 9.38 \\
\hline
\end{tabular}


Table S8. Experimental molar compositions of the liquid $(x)$ phase and predicted molar composition of the vapor $(y)$ phase and activity coefficient $(\gamma)$ using the UNIFAC model for the ternary fragrance mixtures.

\begin{tabular}{|c|c|c|c|c|c|c|c|c|c|c|c|}
\hline \multicolumn{3}{|l|}{ DPG } & \multicolumn{3}{|l|}{ Limonene } & \multicolumn{3}{|l|}{$\alpha$-Pinene } & \multicolumn{3}{|c|}{ Linalyl acetate } \\
\hline$x$ & $y$ & $\gamma$ & $x$ & $y$ & $\gamma$ & $x$ & $y$ & $\gamma$ & $x$ & $y$ & $\gamma$ \\
\hline $7.46 \times 10^{-1}$ & $6.72 \times 10^{-3}$ & 1.16 & $9.46 \times 10^{-2}$ & $2.95 \times 10^{-1}$ & 6.98 & $9.18 \times 10^{-2}$ & $6.86 \times 10^{-1}$ & 6.69 & $6.71 \times 10^{-2}$ & $1.24 \times 10^{-2}$ & 5.76 \\
\hline $8.40 \times 10^{-1}$ & $7.98 \times 10^{-3}$ & 1.06 & $5.80 \times 10^{-2}$ & $2.95 \times 10^{-1}$ & 9.85 & $5.84 \times 10^{-2}$ & $6.84 \times 10^{-1}$ & 9.09 & $4.37 \times 10^{-2}$ & $1.37 \times 10^{-2}$ & 8.45 \\
\hline $8.86 \times 10^{-1}$ & $9.70 \times 10^{-3}$ & 1.03 & $4.24 \times 10^{-2}$ & $3.04 \times 10^{-1}$ & 11.75 & $4.14 \times 10^{-2}$ & $6.72 \times 10^{-1}$ & 10.63 & $3.06 \times 10^{-2}$ & $1.39 \times 10^{-2}$ & 10.32 \\
\hline $9.22 \times 10^{-1}$ & $1.25 \times 10^{-2}$ & 1.01 & $2.91 \times 10^{-2}$ & $3.05 \times 10^{-1}$ & 13.57 & $2.88 \times 10^{-2}$ & $6.69 \times 10^{-1}$ & 12.07 & $2.02 \times 10^{-2}$ & $1.37 \times 10^{-2}$ & 12.16 \\
\hline $9.46 \times 10^{-1}$ & $1.70 \times 10^{-2}$ & 1.01 & $1.96 \times 10^{-2}$ & $3.00 \times 10^{-1}$ & 14.96 & $1.99 \times 10^{-2}$ & $6.68 \times 10^{-1}$ & 13.16 & $1.43 \times 10^{-2}$ & $1.43 \times 10^{-2}$ & 13.59 \\
\hline $9.65 \times 10^{-1}$ & $2.46 \times 10^{-2}$ & 1.00 & $1.31 \times 10^{-2}$ & $3.09 \times 10^{-1}$ & 16.14 & $1.27 \times 10^{-2}$ & $6.52 \times 10^{-1}$ & 14.08 & $9.30 \times 10^{-3}$ & $1.45 \times 10^{-2}$ & 14.83 \\
\hline $9.85 \times 10^{-1}$ & $5.21 \times 10^{-2}$ & 1.00 & $5.68 \times 10^{-3}$ & $3.02 \times 10^{-1}$ & 17.53 & $5.51 \times 10^{-3}$ & $6.32 \times 10^{-1}$ & 15.15 & $4.03 \times 10^{-3}$ & $1.43 \times 10^{-2}$ & 16.31 \\
\hline $9.93 \times 10^{-1}$ & $1.04 \times 10^{-1}$ & 1.00 & $2.56 \times 10^{-3}$ & $2.79 \times 10^{-1}$ & 18.13 & $2.57 \times 10^{-3}$ & $6.03 \times 10^{-1}$ & 15.61 & $1.92 \times 10^{-3}$ & $1.41 \times 10^{-2}$ & 16.96 \\
\hline $9.97 \times 10^{-1}$ & $1.90 \times 10^{-1}$ & 1.00 & $1.25 \times 10^{-3}$ & $2.52 \times 10^{-1}$ & 18.41 & $1.26 \times 10^{-3}$ & $5.45 \times 10^{-1}$ & 15.82 & $9.43 \times 10^{-4}$ & $1.28 \times 10^{-2}$ & 17.26 \\
\hline \multicolumn{3}{|l|}{ DPG } & \multicolumn{3}{|l|}{ Limonene } & \multicolumn{3}{|l|}{$p$-Cymene } & \multicolumn{3}{|l|}{ Linalool } \\
\hline$x$ & $y$ & $\gamma$ & $x$ & $y$ & $\gamma$ & $x$ & $y$ & $\gamma$ & $x$ & $y$ & $\gamma$ \\
\hline $7.36 \times 10^{-1}$ & $1.27 \times 10^{-2}$ & 1.12 & $8.99 \times 10^{-2}$ & $6.06 \times 10^{-1}$ & 7.67 & $9.29 \times 10^{-2}$ & $3.69 \times 10^{-1}$ & 4.64 & $8.08 \times 10^{-2}$ & $1.29 \times 10^{-2}$ & 1.69 \\
\hline $8.31 \times 10^{-1}$ & $1.55 \times 10^{-2}$ & 1.05 & $5.84 \times 10^{-2}$ & $6.19 \times 10^{-1}$ & 10.38 & $6.04 \times 10^{-2}$ & $3.54 \times 10^{-1}$ & 5.89 & $5.04 \times 10^{-2}$ & $1.15 \times 10^{-2}$ & 2.07 \\
\hline $8.75 \times 10^{-1}$ & $1.87 \times 10^{-2}$ & 1.03 & $4.30 \times 10^{-2}$ & $6.20 \times 10^{-1}$ & 12.03 & $4.54 \times 10^{-2}$ & $3.50 \times 10^{-1}$ & 6.61 & $3.68 \times 10^{-2}$ & $1.09 \times 10^{-2}$ & 2.30 \\
\hline $9.19 \times 10^{-1}$ & $2.60 \times 10^{-2}$ & 1.01 & $2.76 \times 10^{-2}$ & $6.22 \times 10^{-1}$ & 14.03 & $2.92 \times 10^{-2}$ & $3.41 \times 10^{-1}$ & 7.47 & $2.38 \times 10^{-2}$ & $1.06 \times 10^{-2}$ & 2.58 \\
\hline $9.45 \times 10^{-1}$ & $3.48 \times 10^{-2}$ & 1.01 & $1.95 \times 10^{-2}$ & $6.27 \times 10^{-1}$ & 15.31 & $2.00 \times 10^{-2}$ & $3.28 \times 10^{-1}$ & 8.01 & $1.60 \times 10^{-2}$ & $9.94 \times 10^{-3}$ & 2.75 \\
\hline $9.62 \times 10^{-1}$ & $4.89 \times 10^{-2}$ & 1.00 & $1.31 \times 10^{-2}$ & $6.19 \times 10^{-1}$ & 16.27 & $1.35 \times 10^{-2}$ & $3.22 \times 10^{-1}$ & 8.40 & $1.17 \times 10^{-2}$ & $1.06 \times 10^{-2}$ & 2.88 \\
\hline $9.84 \times 10^{-1}$ & $1.05 \times 10^{-1}$ & 1.00 & $5.40 \times 10^{-3}$ & $5.85 \times 10^{-1}$ & 17.64 & $5.58 \times 10^{-3}$ & $2.99 \times 10^{-1}$ & 8.96 & $4.85 \times 10^{-3}$ & $9.84 \times 10^{-3}$ & 3.07 \\
\hline $9.93 \times 10^{-1}$ & $2.02 \times 10^{-1}$ & 1.00 & $2.47 \times 10^{-3}$ & $5.24 \times 10^{-1}$ & 18.20 & $2.56 \times 10^{-3}$ & $2.66 \times 10^{-1}$ & 9.19 & $2.13 \times 10^{-3}$ & $8.40 \times 10^{-3}$ & 3.14 \\
\hline $9.96 \times 10^{-1}$ & $3.26 \times 10^{-1}$ & 1.00 & $1.28 \times 10^{-3}$ & $4.42 \times 10^{-1}$ & 18.43 & $1.32 \times 10^{-3}$ & $2.24 \times 10^{-1}$ & 9.28 & $1.10 \times 10^{-3}$ & $7.08 \times 10^{-3}$ & 3.18 \\
\hline \multicolumn{3}{|l|}{ DPG } & \multicolumn{3}{|l|}{ Limonene } & \multicolumn{3}{|c|}{ Ethyl acetate } & \multicolumn{3}{|c|}{ Benzyl acetate } \\
\hline$x$ & $y$ & $\gamma$ & $x$ & $y$ & $\gamma$ & $x$ & $y$ & $\gamma$ & $x$ & $y$ & $\gamma$ \\
\hline $6.78 \times 10^{-1}$ & $1.01 \times 10^{-3}$ & 1.13 & $1.10 \times 10^{-1}$ & $6.74 \times 10^{-2}$ & 8.08 & $1.34 \times 10^{-1}$ & $9.29 \times 10^{-1}$ & 1.51 & $7.89 \mathrm{E}-02$ & $2.38 \times 10^{-3}$ & 3.71 \\
\hline $8.00 \times 10^{-1}$ & $1.58 \times 10^{-3}$ & 1.04 & $6.90 \times 10^{-2}$ & $8.30 \times 10^{-2}$ & 11.10 & $8.19 \times 10^{-2}$ & $9.13 \times 10^{-1}$ & 1.70 & $4.96 \mathrm{E}-02$ & $2.73 \times 10^{-3}$ & 4.77 \\
\hline $8.45 \times 10^{-1}$ & $2.01 \times 10^{-3}$ & 1.03 & $5.22 \times 10^{-2}$ & $8.72 \times 10^{-2}$ & 12.51 & $6.32 \times 10^{-2}$ & $9.08 \times 10^{-1}$ & 1.78 & $3.99 \mathrm{E}-02$ & $2.97 \times 10^{-3}$ & 5.24 \\
\hline $8.96 \times 10^{-1}$ & $2.94 \times 10^{-3}$ & 1.01 & $3.45 \times 10^{-2}$ & $9.18 \times 10^{-2}$ & 14.31 & $4.27 \times 10^{-2}$ & $9.02 \times 10^{-1}$ & 1.88 & $2.68 \mathrm{E}-02$ & $3.10 \times 10^{-3}$ & 5.83 \\
\hline $9.28 \times 10^{-1}$ & $4.22 \times 10^{-3}$ & 1.01 & $2.46 \times 10^{-2}$ & $9.90 \times 10^{-2}$ & 15.52 & $2.94 \times 10^{-2}$ & $8.94 \times 10^{-1}$ & 1.94 & $1.80 \mathrm{E}-02$ & $3.10 \times 10^{-3}$ & 6.23 \\
\hline $9.50 \times 10^{-1}$ & $5.92 \times 10^{-3}$ & 0 & $1.70 \times 10^{-2}$ & $9.99 \times 10^{-2}$ & 16.42 & $2.08 \times 10^{-2}$ & $8.91 \times 10^{-1}$ & 1.98 & 23E-02 & $3.05 \times 10^{-3}$ & 52 \\
\hline
\end{tabular}




\begin{tabular}{llllllllllll}
$9.79 \times 10^{-1}$ & $1.40 \times 10^{-2}$ & 1.00 & $7.13 \times 10^{-3}$ & $1.03 \times 10^{-1}$ & 17.70 & $8.71 \times 10^{-3}$ & $8.80 \times 10^{-1}$ & 2.04 & $5.13 \mathrm{E}-03$ & $3.10 \times 10^{-3}$ & 6.93 \\
$9.91 \times 10^{-1}$ & $3.13 \times 10^{-2}$ & 1.00 & $3.20 \times 10^{-3}$ & $1.06 \times 10^{-1}$ & 18.24 & $3.79 \times 10^{-3}$ & $8.60 \times 10^{-1}$ & 2.07 & $2.30 \mathrm{E}-03$ & $3.15 \times 10^{-3}$ & 7.09 \\
$9.95 \times 10^{-1}$ & $6.14 \times 10^{-2}$ & 1.00 & $1.58 \times 10^{-3}$ & $1.03 \times 10^{-1}$ & 18.46 & $1.87 \times 10^{-3}$ & $8.32 \times 10^{-1}$ & 2.08 & $1.13 \mathrm{E}-03$ & $3.07 \times 10^{-3}$ & 7.16 \\
\hline
\end{tabular}

Table S9. Experimental molar compositions of the liquid (x) phase and predicted composition of the vapor (y) phase and activity coefficient ( $\gamma$ ) using the UNIFAC model for the quaternary fragrance mixtures.

\begin{tabular}{|c|c|c|c|c|c|c|c|c|c|c|c|c|c|c|}
\hline \multicolumn{3}{|l|}{ DPG } & \multicolumn{3}{|l|}{ Limonene } & \multicolumn{3}{|l|}{$\alpha$-Pinene } & \multicolumn{3}{|l|}{$p$-Cymene } & \multicolumn{3}{|c|}{ Linalyl acetate } \\
\hline$x$ & $y$ & $\gamma$ & $x$ & $y$ & $\gamma$ & $x$ & $y$ & $\gamma$ & $x$ & $y$ & $\gamma$ & $x$ & $y$ & $\gamma$ \\
\hline $7.57 \times 10^{-1}$ & $7.60 \times 10^{-3}$ & 1.13 & $6.31 \times 10^{-2}$ & $2.41 \times 10^{-1}$ & 7.51 & $6.57 \times 10^{-2}$ & $5.97 \times 10^{-1}$ & 7.13 & $6.33 \times 10^{-2}$ & $1.42 \times 10^{-1}$ & 4.53 & $5.14 \times 10^{-2}$ & $1.17 \times 10^{-2}$ & 6.18 \\
\hline $8.40 \times 10^{-1}$ & $9.07 \times 10^{-3}$ & 1.05 & $3.97 \times 10^{-2}$ & $2.36 \times 10^{-1}$ & 10.13 & $4.45 \times 10^{-2}$ & $6.09 \times 10^{-1}$ & 9.31 & $4.05 \times 10^{-2}$ & $1.33 \times 10^{-1}$ & 5.74 & $3.52 \times 10^{-2}$ & $1.29 \times 10^{-2}$ & 8.67 \\
\hline $8.80 \times 10^{-1}$ & $1.10 \times 10^{-2}$ & 1.03 & $3.03 \times 10^{-2}$ & $2.48 \times 10^{-1}$ & 11.75 & $3.17 \times 10^{-2}$ & $5.86 \times 10^{-1}$ & 10.63 & $3.22 \times 10^{-2}$ & $1.41 \times 10^{-1}$ & 6.47 & $2.57 \times 10^{-2}$ & $1.32 \times 10^{-2}$ & 10.27 \\
\hline $9.22 \times 10^{-1}$ & $1.51 \times 10^{-2}$ & 1.01 & $2.01 \times 10^{-2}$ & $2.57 \times 10^{-1}$ & 13.74 & $2.03 \times 10^{-2}$ & $5.75 \times 10^{-1}$ & 12.22 & $2.09 \times 10^{-2}$ & $1.39 \times 10^{-1}$ & 7.33 & $1.71 \times 10^{-2}$ & $1.41 \times 10^{-2}$ & 12.30 \\
\hline $9.45 \times 10^{-1}$ & $2.11 \times 10^{-2}$ & 1.01 & $1.37 \times 10^{-2}$ & $2.61 \times 10^{-1}$ & 15.05 & $1.32 \times 10^{-2}$ & $5.57 \times 10^{-1}$ & 13.25 & $1.48 \times 10^{-2}$ & $1.45 \times 10^{-1}$ & 7.89 & $1.29 \times 10^{-2}$ & $1.61 \times 10^{-2}$ & 13.65 \\
\hline $9.66 \times 10^{-1}$ & $2.96 \times 10^{-2}$ & 1.00 & $8.87 \times 10^{-3}$ & $2.54 \times 10^{-1}$ & 16.31 & $9.24 \times 10^{-3}$ & $5.75 \times 10^{-1}$ & 14.213 & $8.90 \times 10^{-3}$ & $1.28 \times 10^{-1}$ & 8.41 & $7.23 \times 10^{-3}$ & $1.37 \times 10^{-2}$ & 15.00 \\
\hline $9.86 \times 10^{-1}$ & $6.57 \times 10^{-2}$ & 1.00 & $3.64 \times 10^{-3}$ & $2.46 \times 10^{-1}$ & 17.66 & $3.80 \times 10^{-3}$ & $5.53 \times 10^{-1}$ & 15.25 & $3.65 \times 10^{-3}$ & $1.22 \times 10^{-1}$ & 8.97 & $2.97 \times 10^{-3}$ & $1.34 \times 10^{-2}$ & 16.45 \\
\hline $9.93 \times 10^{-1}$ & $1.17 \times 10^{-1}$ & 1.00 & $1.80 \times 10^{-3}$ & $2.22 \times 10^{-1}$ & 18.14 & $2.02 \times 10^{-3}$ & $5.36 \times 10^{-1}$ & 15.62 & $1.84 \times 10^{-3}$ & $1.11 \times 10^{-1}$ & 9.16 & $1.60 \times 10^{-3}$ & $1.33 \times 10^{-2}$ & 16.97 \\
\hline $9.95 \times 10^{-1}$ & $1.75 \times 10^{-1}$ & 1.00 & $1.12 \times 10^{-3}$ & $2.08 \times 10^{-1}$ & 18.34 & $1.26 \times 10^{-3}$ & $5.01 \times 10^{-1}$ & 15.77 & $1.15 \times 10^{-3}$ & $1.04 \times 10^{-1}$ & 9.25 & $9.97 \times 10^{-4}$ & $1.24 \times 10^{-2}$ & 17.18 \\
\hline DPG & & & Limonene & & & $\alpha$-Pinene & & & $p$-Cymene & & & Linalool & & \\
\hline$x$ & $y$ & $\gamma$ & $x$ & $y$ & $\gamma$ & $x$ & $y$ & $\gamma$ & $x$ & $y$ & $\gamma$ & $x$ & $y$ & $\gamma$ \\
\hline $7.61 \times 10^{-1}$ & $7.36 \times 10^{-3}$ & 1.10 & $6.13 \times 10^{-2}$ & $2.52 \times 10^{-1}$ & 8.17 & $6.12 \times 10^{-2}$ & $5.88 \times 10^{-1}$ & 7.63 & $6.17 \times 10^{-2}$ & $1.47 \times 10^{-1}$ & 4.87 & $5.49 \times 10^{-2}$ & $5.25 \times 10^{-3}$ & 1.77 \\
\hline $8.43 \times 10^{-1}$ & $9.12 \times 10^{-3}$ & 1.04 & $4.07 \times 10^{-2}$ & $2.60 \times 10^{-1}$ & 10.73 & $4.03 \times 10^{-2}$ & $5.86 \times 10^{-1}$ & 9.75 & $4.00 \times 10^{-2}$ & $1.40 \times 10^{-1}$ & 6.04 & $3.62 \times 10^{-2}$ & $4.93 \times 10^{-3}$ & 2.13 \\
\hline $8.81 \times 10^{-1}$ & $1.11 \times 10^{-2}$ & 1.02 & $2.96 \times 10^{-2}$ & $2.56 \times 10^{-1}$ & 12.26 & $3.01 \times 10^{-2}$ & $5.85 \times 10^{-1}$ & 11.00 & $3.08 \times 10^{-2}$ & $1.42 \times 10^{-1}$ & 6.71 & $2.81 \times 10^{-2}$ & $4.99 \times 10^{-3}$ & 2.34 \\
\hline $9.20 \times 10^{-1}$ & $1.47 \times 10^{-2}$ & 1.01 & $2.09 \times 10^{-2}$ & $2.67 \times 10^{-1}$ & 13.99 & $2.06 \times 10^{-2}$ & $5.81 \times 10^{-1}$ & 12.38 & $2.01 \times 10^{-2}$ & $1.33 \times 10^{-1}$ & 7.45 & $1.86 \times 10^{-2}$ & $4.70 \times 10^{-3}$ & 2.58 \\
\hline $9.44 \times 10^{-1}$ & $1.99 \times 10^{-2}$ & 1.01 & $1.46 \times 10^{-2}$ & $2.68 \times 10^{-1}$ & 15.26 & $1.42 \times 10^{-2}$ & $5.72 \times 10^{-1}$ & 13.38 & $1.45 \times 10^{-2}$ & $1.36 \times 10^{-1}$ & 7.98 & $1.25 \times 10^{-2}$ & $4.43 \times 10^{-3}$ & 2.75 \\
\hline $9.66 \times 10^{-1}$ & $3.07 \times 10^{-2}$ & 1.00 & $8.74 \times 10^{-3}$ & $2.63 \times 10^{-1}$ & 16.50 & $8.72 \times 10^{-3}$ & $5.69 \times 10^{-1}$ & 14.35 & $8.80 \times 10^{-3}$ & $1.33 \times 10^{-1}$ & 8.50 & $7.82 \times 10^{-3}$ & $4.47 \times 10^{-3}$ & 2.92 \\
\hline $9.86 \times 10^{-1}$ & $6.80 \times 10^{-2}$ & 1.00 & $3.62 \times 10^{-3}$ & $2.54 \times 10^{-1}$ & 17.74 & $3.61 \times 10^{-3}$ & $5.47 \times 10^{-1}$ & 15.30 & $3.65 \times 10^{-3}$ & $1.26 \times 10^{-1}$ & 9.00 & $3.24 \times 10^{-3}$ & $4.26 \times 10^{-3}$ & 3.08 \\
\hline $9.93 \times 10^{-1}$ & $1.21 \times 10^{-1}$ & 1.00 & $1.90 \times 10^{-3}$ & $2.42 \times 10^{-1}$ & 18.18 & $1.88 \times 10^{-3}$ & $5.16 \times 10^{-1}$ & 15.64 & $1.87 \times 10^{-3}$ & $1.17 \times 10^{-1}$ & 9.18 & $1.69 \times 10^{-3}$ & $4.00 \times 10^{-3}$ & 3.14 \\
\hline $9.96 \times 10^{-1}$ & $2.17 \times 10^{-1}$ & 1.00 & $9.36 \times 10^{-4}$ & $2.16 \times 10^{-1}$ & 18.43 & $9.28 \times 10^{-4}$ & $4.59 \times 10^{-1}$ & 15.83 & $9.21 \times 10^{-4}$ & $1.04 \times 10^{-1}$ & 9.28 & $8.32 \times 10^{-4}$ & $3.56 \times 10^{-3}$ & 3.18 \\
\hline DPG & & & Limonene & & & $p$-Cymene & & & Ethyl acet & & & Benzyl ace & tate & \\
\hline$x$ & $y$ & $\gamma$ & $x$ & $y$ & $\gamma$ & $x$ & $y$ & $\gamma$ & $x$ & $y$ & $\gamma$ & $x$ & $y$ & $\gamma$ \\
\hline $7.19 \times 10^{-1}$ & $1.43 \times 10^{-3}$ & 1.10 & $5.95 \times 10^{-2}$ & $5.49 \times 10^{-2}$ & 8.85 & $6.10 \times 10^{-2}$ & $3.11 \times 10^{-2}$ & 5.03 & $9.24 \times 10^{-2}$ & $9.10 \times 10^{-1}$ & 1.56 & $6.83 \times 10^{-2}$ & $3.02 \times 10^{-3}$ & 3.98 \\
\hline
\end{tabular}




\begin{tabular}{|c|c|c|c|c|c|c|c|c|c|c|c|c|c|c|}
\hline $8.15 \times 10^{-1}$ & $2.05 \times 10^{-3}$ & 1.04 & $3.88 \times 10^{-2}$ & $6.20 \times 10^{-2}$ & 11.46 & $3.98 \times 10^{-2}$ & $3.38 \times 10^{-2}$ & 6.25 & $6.18 \times 10^{-2}$ & $8.99 \times 10^{-1}$ & 1.72 & $4.49 \times 10^{-2}$ & $3.26 \times 10^{-3}$ & 4.89 \\
\hline $8.58 \times 10^{-1}$ & $2.66 \times 10^{-3}$ & 1.02 & $3.01 \times 10^{-2}$ & $6.73 \times 10^{-2}$ & 12.82 & $3.06 \times 10^{-2}$ & $3.56 \times 10^{-2}$ & 6.86 & $4.70 \times 10^{-2}$ & $8.91 \times 10^{-1}$ & 1.80 & $3.48 \times 10^{-2}$ & $3.45 \times 10^{-3}$ & 5.35 \\
\hline $9.05 \times 10^{-1}$ & $3.91 \times 10^{-3}$ & 1.01 & $2.02 \times 10^{-2}$ & $7.21 \times 10^{-2}$ & 14.54 & $2.07 \times 10^{-2}$ & $3.77 \times 10^{-2}$ & 7.62 & $3.14 \times 10^{-2}$ & $8.83 \times 10^{-1}$ & 1.89 & $2.26 \times 10^{-2}$ & $3.50 \times 10^{-3}$ & 5.92 \\
\hline $9.34 \times 10^{-1}$ & $5.56 \times 10^{-3}$ & 1.00 & $1.38 \times 10^{-2}$ & $7.40 \times 10^{-2}$ & 15.71 & $1.43 \times 10^{-2}$ & $3.84 \times 10^{-2}$ & 8.13 & $2.19 \times 10^{-2}$ & $8.79 \times 10^{-1}$ & 1.95 & $1.56 \times 10^{-2}$ & $3.55 \times 10^{-3}$ & 6.30 \\
\hline $9.59 \times 10^{-1}$ & $8.95 \times 10^{-3}$ & 1.00 & $8.68 \times 10^{-3}$ & $7.77 \times 10^{-2}$ & 16.76 & $8.89 \times 10^{-3}$ & $3.97 \times 10^{-2}$ & 8.58 & $1.35 \times 10^{-2}$ & $8.70 \times 10^{-1}$ & 2.00 & $9.96 \times 10^{-3}$ & $3.76 \times 10^{-3}$ & 6.63 \\
\hline $9.83 \times 10^{-1}$ & $2.11 \times 10^{-2}$ & 1.00 & $3.61 \times 10^{-3}$ & $7.95 \times 10^{-2}$ & 17.86 & $3.70 \times 10^{-3}$ & $4.01 \times 10^{-2}$ & 9.04 & $5.60 \times 10^{-3}$ & $8.56 \times 10^{-1}$ & 2.05 & $4.15 \times 10^{-3}$ & $3.80 \times 10^{-3}$ & 6.98 \\
\hline $9.92 \times 10^{-1}$ & $4.27 \times 10^{-2}$ & 1.00 & $1.70 \times 10^{-3}$ & $7.69 \times 10^{-2}$ & 18.28 & $1.75 \times 10^{-3}$ & $3.87 \times 10^{-2}$ & 9.22 & $2.72 \times 10^{-3}$ & $8.38 \times 10^{-1}$ & 2.07 & $1.97 \times 10^{-3}$ & $3.69 \times 10^{-3}$ & 7.11 \\
\hline $9.96 \times 10^{-1}$ & $7.46 \times 10^{-2}$ & 1.00 & $9.41 \times 10^{-4}$ & $7.47 \times 10^{-2}$ & 18.46 & $9.66 \times 10^{-4}$ & $3.76 \times 10^{-2}$ & 9.29 & $1.50 \times 10^{-3}$ & $8.10 \times 10^{-1}$ & 2.08 & $1.09 \times 10^{-3}$ & $3.57 \times 10^{-3}$ & 7.16 \\
\hline
\end{tabular}

